\title{
Shielding circuits with groups
}

\author{
Eric Miles* $\quad$ Emanuele Viola*
}

February 24, 2014

\begin{abstract}
We show how to efficiently compile any given circuit $C$ into a leakage-resilient circuit $\widehat{C}$ such that any function on the wires of $\widehat{C}$ that leaks information during a computation $\widehat{C}(x)$ yields advantage in computing the product of $|\widehat{C}|^{\Omega(1)}$ elements of the alternating group $A_{u}$. Our construction resists $\mathrm{NC}^{1}$ leakage assuming $\mathrm{L} \neq \mathrm{NC}^{1}$, as was conjectured here and proven later [Miles, ITCS '14]. Also, in combination with new compression bounds for $A_{u}$ products obtained here, $\widehat{C}$ withstands leakage from virtually any class of functions against which average-case lower bounds are known. This includes communication protocols, and $\mathrm{AC}^{0}$ circuits augmented with few arbitrary symmetric gates. In addition, we extend the construction to the multi-query setting by relying on a simple secure hardware component.

We build on Barrington's theorem [JCSS '89] and on the previous leakage-resilient constructions by Ishai et al. [Crypto '03] and Faust et al. [Eurocrypt '10]. Our construction exploits properties of $A_{u}$ beyond what is sufficient for Barrington's theorem.
\end{abstract}

${ }^{*}$ Supported by NSF grants CCF-0845003 and CCF-1319206. Email: \{enmiles, viola\}@ccs .neu.edu 


\section{Introduction}

Motivated by successful attacks on cryptographic hardware, a recent, exciting line of work known as leakage-resilient cryptography considers models in which the adversary obtains more information from cryptographic algorithms than just their input/output behavior. A general goal in this area is to compile any circuit into a new "shielded" circuit such that any attack exploiting this extra information can in fact be carried out just using input/output access (and hence does not succeed under standard hardness assumptions). However, the seminal impossibility result on obfuscation $\left[\mathrm{BGI}^{+} 01\right]$ implies that one cannot shield circuits against an attack that obtains just one extra bit of information about the circuit, if this bit is computed as an arbitrary efficient leakage function of the wires of the circuit. More specifically it is sufficient that the leakage function is powerful enough to evaluate the shielded circuit on its own description. (There is in fact a close connection between obfuscation and leakage resilience, see e.g. the discussion in [Rot12]).

Still, this negative result does not necessarily hinder the scope of a theoretical study of leakage-resilient cryptography, because in practice this extra information is quite difficult to obtain and is typically limited to some simple-to-compute functions such as the Hamming weight of the bits carried on the wires. Thus, it makes sense to focus our attention on attacks where the extra information is obtained from the circuit by evaluating a computationally restricted leakage function.

One line of works considers a model that has become known as "only computation leaks", after Micali and Reyzin [MR04]. In this model, the compiled circuit is partitioned (by the compiler) into topologically ordered sets of wires, i.e. so that the value of each wire depends only on wires in its set or in sets preceding it. The leakage function then operates separately on each set in the partition. Ishai, Sahai, and Wagner in [ISW03] allow the leakage function to output projections of few of (the values carried on) the wires in each set. Their result is greatly generalized by a series of works [GR10, JV10, DF12, GR12] culminating in the construction by Goldwasser and Rothblum [GR12] which allows any arbitrary function of each set, as long as the function has bounded output length.

In a different direction, Faust et al. $\left[\mathrm{FRR}^{+} 10\right]$ allow leakage functions that are computable by small, bounded-depth circuits with And, Or, and Not gates $\left(\mathrm{AC}^{0}\right)$. In contrast to the previous setting, here the leakage function accesses all wires simultaneously; we refer to this as the "global" leakage model. In the case of an unbounded number of queries from the adversary - so-called "continual leakage" - the compiled circuits in [FRR $\left.{ }^{+} 10\right]$ use a randomized, inputless gate that outputs a bit vector that is uniform up to having parity $=0$. In contrast, the only randomized gates used in [ISW03, GR12] output uniform and independent bits. Gates that produce bits from a distribution other than uniform are referred to in the literature as "secure hardware components". The use of secure hardware in [FRR $\left.{ }^{+} 10\right]$ was removed by Rothblum [Rot12] at the expense of introducing a computational assumption.

\subsection{Our results}

In this work we give a new construction of a leakage-resilient compiler. In the multi-query setting, our construction uses a secure hardware component similar to the one in $\left[\mathrm{FRR}^{+} 10\right]$. Our construction: 
1. is secure against global $\mathrm{NC}^{1}$ leakage assuming $\mathrm{NC}^{1} \neq \mathrm{L}$, as was conjectured here and proved later by Miles [Mil14].

2. is candidate to having security in the OCL model; we are not aware of any other construction that is candidate to having security simultaneously in both the global and OCL leakage models.

3. is unconditionally secure against global leakage from natural, well-studied classes of functions that break nearly all previous constructions; for example, it resists leakage from parity and inner product which break [ISW03, FRR ${ }^{+}$10, DF12, GR12, Rot12].

For simplicity, we first focus on the setting where the adversary makes a single query to the circuit, and we do not use any secure hardware. Defined next, our compiler is randomized and takes two inputs: a circuit $C:\{0,1\}^{n} \times\{0,1\}^{n} \rightarrow\{0,1\}^{n}$, and a value $k \in\{0,1\}^{n}$ for $C^{\prime}$ 's second input. It outputs a circuit $\widehat{C}:\{0,1\}^{n} \rightarrow\{0,1\}^{n}$ that is functionally equivalent to $C(\cdot, k)$ (we choose to omit a $k$ subscript though $\widehat{C}$ depends on $k$ ). The only parts of $\widehat{C}$ that depend on $k$ and the random coins are the values of its constant gates; the rest is determined by $C$. The adversary depends on $C$ and thus knows everything about $\widehat{C}$ except the values of certain constant gates. The adversary then selects both an input $x$ to the circuit and a leakage function to be evaluated on the wires of the circuit. The requirement that the adversary "learns nothing" from the output of the leakage function is formalized by providing an efficient simulator $S$. $S$ sees only the input $x$ and output $\widehat{C}(x)$ of the circuit, as well as the circuit $C$ which is assumed to be public, and produces a set of wire values that is indistinguishable from the real set of wire values by the leakage function. Throughout the paper we will use $|C|$ to denote the number of wires in a circuit $C$, which is the input length of the leakage functions.

Definition 1.1 (Leakage-secure compiler). Let $\operatorname{Comp}(\cdot, \cdot)$ denote a randomized algorithm that takes as input a circuit $C:\{0,1\}^{n} \times\{0,1\}^{n} \rightarrow\{0,1\}^{n}$ and a string $k \in\{0,1\}^{n}$. For a set of functions $\mathcal{L}$, Comp is an $(\mathcal{L}, \epsilon)$-leakage-secure compiler if the following properties hold.

1. (Structure.) For every $C$ and $k, \operatorname{Comp}(C, k)$ outputs a circuit $\widehat{C}:\{0,1\}^{n} \rightarrow\{0,1\}^{n}$ which is completely determined by $C$ except for the values of its constant gates.

2. (Correctness.) For every $C$ and $k$ and every $x \in\{0,1\}^{n}, \widehat{C}(x)=C(x, k)$ with probability 1 over the choice of $\widehat{C} \leftarrow \operatorname{Comp}(C, k)$.

3. (Security.) There exists a randomized polynomial-time algorithm $S$ such that for every $C$ and $k$, every $x \in\{0,1\}^{n}$, and every $\ell \in \mathcal{L}$ with domain $\{0,1\}^{|\widehat{C}|}$ :

$$
\Delta(\ell(\widehat{W}), \ell(S(C, x, \widehat{C}(x)))) \leq \epsilon
$$

where $\widehat{W} \in\{0,1\}^{|\widehat{C}|}$ denotes the values carried by the wires of $\widehat{C}(x)$, and the statistical distance $\Delta$ is over the choice of $\widehat{C} \leftarrow \operatorname{Comp}(C, k)$ and the random coins of $S$.

We show how to efficiently compile any circuit $C$ into a leakage-resilient circuit $\widehat{C}$ such that any function on the wires of $\widehat{C}$ that leaks information during some computation $\widehat{C}(x)$ yields advantage in computing iterated group products over the alternating group $A_{u}$, which recall is the group of even permutations of a set of size $u$. (For background on this group, 
see e.g. [KS04, §4.3].) The security of our construction is proved against leakage classes $\mathcal{L}$ for which iterated products over $A_{u}$ are hard in the following sense. As discussed later we exploit specific properties of $A_{u}$, but when possible we present things over any group $G$.

Definition 1.2 ( $\epsilon$-fooled). Let $G$ be a group (whose operation is written multiplicatively). For $\alpha \in G$ and $t \in \mathbb{N}$, let $D_{\alpha}$ denote the uniform distribution over $\left\{\left(x_{1}, \ldots, x_{t}\right) \in G^{t} \mid \prod_{i} x_{i}=\right.$ $\alpha$, and let $U_{G^{t}}$ denote the uniform distribution over $G^{t}$.

Then a set of functions $\mathcal{L}$ is $\epsilon$-fooled by $G^{t}$ if $\Delta\left(\ell\left(D_{\alpha}\right), \ell\left(U_{G^{t}}\right)\right) \leq \epsilon$ for every $\alpha \in G$ and every $\ell \in \mathcal{L}$ with domain $G^{t}$.

We will use the notation $D_{\alpha}, U_{G^{t}}$ throughout the paper. Our security reductions are computable by simple, local (a.k.a. $\mathrm{NC}^{0}$ ) functions.

Definition 1.3 (Local extension). A function $f: G^{t} \rightarrow G^{*}$ is a d-local function if each output element depends on at most $d$ input elements. If $d=O(1)$ as a function of $t$, we simply say local. For a set of functions $\mathcal{L}$, the $d$-local extension of $\mathcal{L}$ is the set of all functions $\ell(f(\cdot))$ where $\ell \in \mathcal{L}$ and $f$ is a $d$-local function.

Note that 1-local functions are also known as projections.

Our compiler is given by the following main theorem.

Theorem 1.4. Let $G$ be a group. For every polynomial-time computable function $t=t(n)$, there is a compiler Comp for which the following holds.

1. For every $C:\{0,1\}^{n} \times\{0,1\}^{n} \rightarrow\{0,1\}^{n}$ and $k \in\{0,1\}^{n}$, $\operatorname{Comp}(C, k)$ runs in time poly $(|C|, t)$ and outputs a circuit $\widehat{C}$ of size $O\left(t^{2} \cdot|C|\right)$ and depth $O(t \cdot \operatorname{depth}(C))$.

2. For every set of functions $\mathcal{L}$ and every $\epsilon>0$, if the 4 -local extension of $\mathcal{L}$ is $\epsilon$-fooled by $G^{t}$ then Comp is an $(\mathcal{L}, \epsilon \cdot t \cdot|C|)$-leakage-secure compiler.

Note that making $t$ smaller reduces the size overhead of $\widehat{C}$, but that larger values of $t$ are necessary to find rich classes $\mathcal{L}$ that are fooled by $G^{t}$.

To instantiate our construction we prove in $\S 3$ that $\left(A_{u}\right)^{t}$ fools a number of well-studied classes of functions (with parameters polynomially related to $t$ ). For all these results we can and will choose $u=5$. One class is that of number-on-forehead multiparty protocols introduced by Chandra, Furst, and Lipton [CFL83], which are formally defined in $\S 3.1$; here our result relies on the long-standing lower bound by Babai, Nisan, and Szegedy [BNS92], whose proof is increasingly streamlined in [CT93, Raz00, VW08]. Another is the class $\mathrm{AC}^{0}$ of bounded-depth And/Or/Not circuits augmented with few gates computing arbitrary symmetric functions, such as parity and majority. This is the richest circuit class for which super-polynomial average-case lower bounds are known [Vio07]. In fact, one can allow few gates whose local extension has low number-on-forehead communication under any partition, such as polynomial threshold functions [Nis93, Vio13]. We also consider the class $\mathrm{TC}^{0}$ of bounded-depth circuits of majority gates; for this class no lower bound is known, and our results rely on the standard complexity assumption $\mathrm{TC}^{0} \neq \mathrm{NC}^{1}$.

The following theorem summarizes the results above. These results can also be seen as giving compression bounds, similar to the work of Dubrov and Ishai [DI06] (see also [HN10, Dru12] and others). In fact, we essentially recover for $A_{5}$-products the parameters 
of the [DI06] compression bound against $\mathrm{AC}^{0}$ (building on their result), and also prove compression bounds against stronger classes.

In the following, $O_{d}(\cdot)$ and $\Omega_{d}(\cdot)$ hide constants that depend only on $d$.

Theorem 1.5. $\left(A_{5}\right)^{t} \epsilon$-fools $\mathcal{L}$ for:

1. $\mathcal{L}=$ number-on-forehead protocols with $s$ parties communicating and outputting $\leq c$ bits, under a specific partition of the input; $\epsilon=2^{c-\Omega\left(t /\left(s^{2} 4^{s}\right)\right)}$.

2. $\mathcal{L}=\mathrm{AC}^{0}$ circuits with depth $\leq d$, size $\leq t^{O_{d}(\log t)}$, an additional $O_{d}\left(\log ^{2} t\right)$ arbitrary symmetric gates, and $t^{0.1}$ bits of output; $\epsilon=t^{-\Omega_{d}(\log t)}$.

3. If $\mathrm{TC}^{0} \neq \mathrm{NC}^{1}$ then for every $k$ and infinitely many $t, \mathcal{L}=\mathrm{TC}^{0}$ circuits with size $\leq t^{k}$ and $k \log t$ bits of output; $\epsilon=t^{-k}$.

4. $\mathcal{L}=\mathrm{AC}^{0}$ circuits with depth $\leq d$, size $\leq 2^{O_{d}\left(t^{(1-\delta) / d}\right)}$, and $t^{\delta}$ bits of output, for any $\delta<1 ; \epsilon=2^{-\Omega_{d}\left(t^{(1-\delta) / d}\right)}$.

The straightforward combination of Theorems 1.4 and 1.5 gives an $(\mathcal{L}, \epsilon)$-secure compiler for the circuit classes listed in items 2-4 of the latter, choosing $t=|C|$ for the following corollary. The combination is less straightforward for protocols, which are not closed under composition with arbitrary local functions. We obtain item 1 of the following corollary by showing (in §4) that the local extension of a number-in-hand protocol is computable by a number-on-forehead protocol.

Corollary 1.6. There is a single efficient compiler Comp, outputting a circuit $\widehat{C}$ of size $|\widehat{C}|=O\left(|C|^{3}\right)$, that is an $(\mathcal{L}, \epsilon)$-leakage secure compiler for each of the following.

1. $\mathcal{L}=$ number-in-hand protocols with s parties communicating and outputting $\leq \delta \cdot|\widehat{C}|^{1 / 3}$ bits, for a fixed $\delta>0$ and a fixed partition of $\widehat{C}$ into $s=O(1)$ sets; $\epsilon=2^{-\Omega\left(|\widehat{C}|^{1 / 3}\right)}$.

2. $\mathcal{L}=\mathrm{AC}^{0}$ circuits with depth $\leq d$, size $\leq|\widehat{C}|^{O_{d}(\log |\widehat{C}|)}$, an additional $O_{d}\left(\log ^{2}|\widehat{C}|\right)$ arbitrary symmetric gates, and $|\widehat{C}|^{0.01}$ bits of output; $\epsilon=|\widehat{C}|^{-\Omega_{d}(\log |\widehat{C}|)}$.

3. If $\mathrm{TC}^{0} \neq \mathrm{NC}^{1}$ then for every $k$ and infinitely many $|C|, \mathcal{L}=\mathrm{TC}^{0}$ circuits with size $\leq|\widehat{C}|^{k}$ and $k \log |\widehat{C}|$ bits of output; $\epsilon=|\widehat{C}|^{-k}$.

4. $\mathcal{L}=\mathrm{AC}^{0}$ circuits with depth $\leq d$, size $\leq 2^{O_{d}\left(\mid \widehat{C}^{(1-\delta) / 3 d}\right)}$, and $|\widehat{C}|^{\delta / 3}$ bits of output, for any $\delta<1 ; \epsilon=2^{-\Omega_{d}\left(|\widehat{C}|^{(1-\delta) / 3 d}\right)}$.

In particular, our construction resists leakage from functions such as parity, majority, inner product, and polynomial thresholds. Besides being well-studied, these functions break most previous constructions. For example, inner product breaks [DF12, GR12], and parity breaks [ISW03, $\mathrm{FRR}^{+} 10$, Rot12]. Also, small $\mathrm{TC}^{0}$ circuits can be shown to break at least one instantiation of the construction [JV10] using the fact that such circuits may compute division, cf. [All01]. In fact, we are only aware of one construction that is not easily broken in $\mathrm{TC}^{0}$. This is the construction [GR10] which relies on the Decisional-Diffie-Hellman assumption. It is broken by any leakage function that can decrypt a certain public-key cryptosystem based on it, but decryption here involves modular exponentiation (to a poly-length exponent); whether this is doable in small depth is an open problem.

Finally, note that the last item shows that we recover the security of $\left[\mathrm{FRR}^{+} 10\right]$ against $\mathrm{AC}^{0}$ leakage functions. 
Security against $\mathrm{NC}^{1}$. We conjecture that our construction is secure even against leakage from functions computable in $\mathrm{NC}^{1}$. Obviously $\left(A_{u}\right)^{t}$ does not fool $\mathrm{NC}^{1}$ circuits when $u=$ $O(1)$, for such circuits can simply compute the product. However it is not clear how such a computation would go when $u=\omega(1)$. Recall (Def. 1.2) that the security of our construction is based on showing that for every $\alpha \in A_{u}$, leakage functions cannot distinguish between a uniform vector and one whose product is fixed to $\alpha$. Thus by an averaging argument and the random self-reducibility of group products, the conjecture would follow from showing that for every $\alpha \mathrm{NC}^{1}$ circuits cannot decide (in the worst case) between vectors with product $\alpha$ and those with product id.

Motivation for this conjecture comes from the work of Cook and McKenzie [CM87] who show that computing products over the symmetric group $S_{u}$ is complete for $\mathrm{L}=$ logarithmic space (this easily extends to $A_{u}$ products). However, the specific decision versions of this problem that they consider are not sufficient for our purposes. If one could use their construction to show that the above " $\alpha$ vs. id" problem is L-complete for every $\alpha$, then the conjecture would hold under the standard assumption $\mathrm{NC}^{1} \neq \mathrm{L}$. (Update: the conjecture was proved in [Mil14].)

Only computation leaks. Next we discuss the security of our construction in the "only computation leaks" (OCL) model due to Micali and Reyzin [MR04]. In this model, the compiler specifies a topologically-ordered partition $P=\left(P_{1}, \ldots, P_{r}\right)$ on the wires of $\widehat{C}$. (Topologically-ordered means that for each $P_{i} \in P$ and each wire $w \in P_{i}$, w's value in $\widehat{C}$ 's computation depends only on wires $w^{\prime}$ such that $w^{\prime} \in P_{j}$ for some $j \leq i$.) Then for each set $P_{i}$ in the partition, the adversary chooses a leakage function to be applied to $P_{i}$ 's wires during the computation $\widehat{C}(x)$ for its chosen input $x$.

The goal in this setting is to tolerate leakage functions that are chosen adaptively for each $P_{i}$ and are computationally unrestricted other than a bound on their output length. The work of Goldwasser and Rothblum [GR12] achieves this with a partition into $O(|C|)$ sets each of size $O\left(t^{\omega}\right)$ and tolerates $\Omega(t)$ bits of leakage per set, where $\omega$ is the matrix multiplication exponent. Furthermore, their construction does not use secure hardware components.

Without the topological ordering requirement, item 1 of Corollary 1.6 gives a partition into $O(1)$ sets each of size $O\left(t^{2} \cdot|C| \cdot \log |G|\right)$ and tolerates $\Omega(t)$ bits of leakage per set. (For this discussion we let $G$ grow asymptotically and explicitly note its size.) Next we refine this into a topologically-ordered partition with $O(t \cdot|C|)$ sets each of size $O(t \log |G|)$. The amount of leakage tolerated depends on the strength of communication lower bounds for number-on-forehead (NOF) protocols, as stated in the following theorem. For $s$-party NOF protocols, we refer to the canonical partition of $x \in G^{t}$ as the one in which player $i$ 's forehead contains $x_{i}, x_{i+s}, \ldots, x_{i+t-s}$.

Theorem 1.7. Assume that 8-party NOF protocols communicating $\leq c$ bits are $\epsilon$-fooled by $G^{t}$ under the canonical partition of $x \in G^{t}$.

Then for each $C$ and $k$ there is a topologically-ordered partition $P$ on $\widehat{C}:=\operatorname{Comp}(C, k)$ containing $O(t \cdot|C|)$ sets each of size $O(t \log |G|)$, such that Comp from Theorem 1.4 is an $(\mathcal{L}, \epsilon \cdot t \cdot|C|)$-leakage secure compiler for $\mathcal{L}=$ all $O C L$ leakage functions that output $\leq \delta \cdot c / t$ bits per set in $P$, where $\delta$ is a constant that depends only on the maximum fanout of $C$. 
Item 1 of Theorem 1.5 gives a lower bound for NOF protocols that achieves $c=\Omega(t)$ and $\epsilon=2^{-\Omega(t)}$ for any constant number of parties. When the group size is constant, this is optimal up to constant factors as the entire input to the NOF protocol has size $t \cdot|G|=O(t)$. Unfortunately, plugging this into Theorem 1.7 does not give anything because $c / t<1$.

In order to use Theorem 1.7 to achieve security in the OCL model, one must prove communication lower bounds that grow with the size of the group. For example one could ask whether, for some $\delta>0$, NOF protocols communicating $c=\delta t \log t$ bits are fooled by $\left(A_{t}\right)^{t}$. By Theorem 1.7, this would give a partition of $\widehat{C}$ into $O(t \cdot|C|)$ sets of size $O\left(t^{2} \log t\right)$ and allow $\Omega(\log t)$ bits of leakage per set. By comparison, recall that [GR12] achieves a partition into $O(|C|)$ sets of size $O\left(t^{\omega}\right)$ and allows $\Omega(t)$ bits of leakage per set.

Interestingly, for groups such as $A_{t}$ whose elements are represented as permutations on a set $[t]$, one can only hope to prove communication lower bounds of the form $c=\Omega(t \log t)$. This is because a protocol that "traces" some point in $[t]$ through the permutations can distinguish any two fixed products with communication $O(t \log t)$. If one could instead obtain an $\Omega\left(t^{2}\right)$ lower bound against NOF protocols computing products over some group $G$ of size $|G|=2^{\Theta(t)}$, this would give a compiler tolerating $\Omega(t)$ bits of leakage per set of size $O\left(t^{2}\right)$, essentially matching [GR12] (modulo the use of secure hardware). Because any group $G$ can be embedded in $S_{|G|}$ by Cayley's theorem, there is always a "tracing" protocol that communicates $O(t \log |G|)=O\left(t^{2}\right)$ bits. (In fact the trivial protocol that communicates its entire input already achieves this bound.) Thus we are posing the question of whether there is any group $G$ for which this is tight.

Multiple queries. We also consider the setting in which the adversary makes multiple, adaptive queries to the circuit $\widehat{C}$. As in the previous setting, each query consists of both an input to the circuit and a leakage function. The adversary is assumed to be computationally unbounded, except for the restriction on the leakage functions. We defer until $\S 5$ the formal definition of security in this setting, but it is a natural extension of Definition 1.1.

If the number of queries $q$ is fixed in advance and known to the compiler, then our construction in Theorem 1.4 can be extended with little difficulty to this setting. The resulting construction increases the size of $\widehat{C}$ by a factor of $O(q)$ and likewise the security degrades by a factor of $q$ (details omitted).

When the number of queries $q$ is not a priori bounded, we adopt the approach of $\left[\mathrm{FRR}^{+} 10\right]$ and augment $\widehat{C}$ with a so-called secure hardware component. In our construction, this component is a randomized, inputless gate that on each execution outputs a sample from $D_{\text {id }}$, where id denotes the identity element of $A_{5}$. We refer to such gates as $D_{\text {id }}$-gates, and any circuit that contains one as a $D_{\mathrm{id}}$-circuit. The complexity of this component is comparable to the one in $\left[\mathrm{FRR}^{+} 10\right]$ which outputs a uniform bit vector with parity 0 . (Secure hardware components are also used in [GR10, JV10], but there the components are not inputless and furthermore the distribution sampled is significantly more complex.)

To prove security in this setting, a slightly stronger property is required of $\left(A_{5}\right)^{t}$ than what is given by Theorem 1.5. Specifically we require that, for every $\ell \in \mathcal{L}$ and every $\ell^{\prime} \in \mathcal{L}$ that is chosen adaptively based on the output of $\ell$, the distribution $\left(\ell(x), \ell^{\prime}(x)\right)$ when $x \leftarrow D_{\alpha}$ has statistical distance $\leq \epsilon$ from the corresponding distribution when $x \leftarrow U_{G^{t}}$. We show in $\S 5.1$ that each of the classes $\mathcal{L}$ listed in Theorem 1.5 has this property; the only difference is 
that for $\mathrm{AC}^{0}$ circuits with symmetric gates, we restrict the output length to $O\left(\log ^{2} t\right)$.

We defer the details for now and simply state our result for multiple queries.

Corollary 1.8. There is a single efficient compiler Comp, outputting a $D_{\mathrm{id}}$-circuit $\widehat{C}$ of size $|\widehat{C}|=O\left(|C|^{3}\right)$, that is a q-query $(\mathcal{L}, \epsilon)$-leakage secure compiler for any $q$ and each of the following.

1. $\mathcal{L}=$ number-in-hand protocols with s parties communicating $\leq \delta \cdot|\widehat{C}|^{1 / 3}$ bits, for a fixed $\delta>0$ and a fixed partition of $\widehat{C}$ into $s=O(1)$ sets; $\epsilon=q \cdot 2^{-\Omega\left(|\widehat{C}|^{1 / 3}\right)}$.

2. $\mathcal{L}=\mathrm{AC}^{0}$ circuits with depth $\leq d$, size $\leq|\widehat{C}|^{O_{d}(\log |\widehat{C}|)}$, an additional $O_{d}\left(\log ^{2}|\widehat{C}|\right)$ arbitrary symmetric gates, and $O_{d}\left(\log ^{2}|\widehat{C}|\right)$ bits of output; $\epsilon=q \cdot|\widehat{C}|^{-\Omega_{d}(\log |\widehat{C}|)}$.

3. If $\mathrm{TC}^{0} \neq \mathrm{NC}^{1}$ then for every $k$ and infinitely many $|C|, \mathcal{L}=\mathrm{TC}^{0}$ circuits with size $\leq|\widehat{C}|^{k}$ and $k \log |\widehat{C}|$ bits of output; $\epsilon=q \cdot|\widehat{C}|^{-k}$.

4. $\mathcal{L}=\mathrm{AC}^{0}$ circuits with depth $\leq d$, size $\leq 2^{O_{d}\left(\mid \widehat{C}^{(1-\delta) / 3 d}\right)}$, and $|\widehat{C}|^{\delta / 3}$ bits of output, for any $\delta<1 ; \epsilon=q \cdot 2^{-\Omega_{d}\left(|\widehat{C}|^{(1-\delta) / 3 d}\right)}$.

Organization. In $\S 2$ we describe our construction and prove the key lemma that enables the proof of Theorem 1.4. In $\S 3$ we show that various computational models are fooled by $\left(A_{5}\right)^{t}$, proving Theorem 1.5. In $\S 4$ we prove Theorem 1.4, Corollary 1.6, and Theorem 1.7. In $\S 5$ we extend our construction to multiple queries.

\section{The construction}

In this section we describe our main construction. Our compiler uses the general framework of the works [ISW03, $\mathrm{FRR}^{+}$10]. In this framework, to every wire of $C$ there corresponds in the compiled circuit $\widehat{C}$ a "bundle" of wires which encode the same information. (In [ISW03, $\mathrm{FRR}^{+}$10] a bit $b$ is encoded by a bundle $x$ whose parity is $b$.) One then uses appropriate gadgets to simulate the computation of $C$ on the bundles. Note the distinction between gates and gadgets in $\widehat{C}$ : gadgets operate on bundles of wires, and are composed of gates that operate on individual wires.

The main differences between our construction and the ones in [ISW03, FRR $\left.{ }^{+} 10\right]$ are in the encoding and in the gadgets. A side-benefit of our gadgets is that they allow for a more modular construction yielding an arguably more intuitive proof of security. Next we describe our encoding, our gadgets, and the proof of security. But first we make some remarks on the group used throughout.

The choice of the group. This work exploits 3 properties of the alternating group $A_{5}$.

(i) It fools various classes in the sense of Definition 1.2 (see Theorem 1.5). We show that this is implied by the fact that every element of $A_{5}$ is a commutator; such groups are known as perfect [HP89].

(ii) It supports Barrington's encoding of $\mathrm{NC}^{1}$ computation [Bar89], which we use in the construction of the NAND gadget below. (This is implied by the group being non-solvable, which in turn is implied by it being perfect.) 
(iii) It has specific elements that support a more efficient encoding of certain functions such as parity, improving on (ii). This is used in Theorem 1.5 to obtain improved parameters and in particular to match the parameters of the previous compression bound in [DI06].

We point out that (i) is not implied by (ii). Indeed, for (ii) the group $S_{5}$ is typically chosen. However $\left(S_{5}\right)^{t}$ does not even (1/2)-fool the 1-local extension of parity, which can compute the sign of the product permutation. This is because the sign of $D_{\alpha}$ always equals the sign of $\alpha$, whereas the sign of $U_{\left(S_{5}\right)^{t}}$ is equidistributed over $\{-1,1\}$.

The group encoding. We encode a bit $b \in\{0,1\}$ by a tuple of elements over a group $G$ as follows. Let id denote $G$ 's identity element, and fix an element id $\neq \alpha \in G$. Then $\left(x_{1}, \ldots, x_{t}\right) \in G^{t}$ encodes $b$ when

$$
\prod_{i} x_{i}= \begin{cases}\text { id } & \text { if } b=0 \\ \alpha & \text { if } b=1\end{cases}
$$

As in [Bar89], we can use any $\alpha$ for which there exists an element $\beta \in G$ such that $\alpha, \beta$, and $\alpha \beta \alpha^{-1} \beta^{-1}$ are in the same conjugacy class. Equivalently, there must exist three elements $\beta, \gamma, \rho \in G$ such that the following two equations hold.

$$
\gamma \alpha \gamma^{-1}=\beta \quad \rho \alpha \beta \alpha^{-1} \beta^{-1} \rho^{-1}=\alpha .
$$

For $G=A_{5}$ and using cycle notation, these values can be set as follows: $\alpha=(12345)$, $\beta=\rho=(14235), \gamma=(12354)$.

For convenience we present the construction over $G$ as opposed to $\{0,1\}$ and using gates for group multiplication and inversion. It is straightforward to obtain a construction over $\{0,1\}$ and any standard basis by implementing group operations via bit operations.

The NAND gadget. We assume without loss of generality that $C$, the circuit input to the compiler, contains only fan-in-2 gates that compute the Nand function. We now describe the NAND gadget that simulates each Nand gate in $C$. Given as input two bundles $x, y \in G^{t}$ with products in $\{$ id, $\alpha\}$, the NAND gadget outputs a bundle $z \in G^{t}$ that encodes the Nand of $x$ and $y$, i.e., that satisfies:

$$
\prod_{i} z= \begin{cases}\text { id } & \text { if } \prod_{i} x_{i}=\prod_{i} y_{i}=\alpha \\ \alpha & \text { otherwise. }\end{cases}
$$

The output bundle $z \in G^{t}$ is computed by the following steps.

1. Set $y \leftarrow\left(\gamma \cdot y_{1}, y_{2}, \ldots, y_{t-1}, y_{t} \cdot \gamma^{-1}\right)$. (This gives $\prod_{i} y_{i} \in\{$ id,$\beta\}$.)

2. Compute $x^{-1}:=\left(x_{t}^{-1}, \ldots, x_{1}^{-1}\right)$ and similarly $y^{-1}$.

3. Compute $\bar{z} \in G^{4 t}$ by concatenating $\left(x, y, x^{-1}, y^{-1}\right)$. (This gives $\prod_{i} \bar{z}_{i} \in\left\{\right.$ id, $\left.\alpha \beta \alpha^{-1} \beta^{-1}\right\}$.)

4. Set $\bar{z} \leftarrow\left(\rho \cdot \bar{z}_{1}, \bar{z}_{2}, \ldots, \bar{z}_{4 t-1}, \bar{z}_{4 t} \cdot \rho^{-1}\right)$. (This gives $\prod_{i} \bar{z}_{i} \in\{$ id,$\alpha\}$.) 
5. Set $\bar{z} \leftarrow\left(\bar{z}_{4 t}^{-1}, \ldots, \bar{z}_{1}^{-1} \cdot \alpha\right)$. (This maintains $\prod_{i} \bar{z}_{i} \in\{\mathrm{id}, \alpha\}$ but if the product in step 4 was $\alpha$ it is now id, and vice versa.)

6. Compute and output $z \in G^{t}$ by multiplying consecutive groups of 4 elements in $\bar{z}$ :

$$
z:=\left(\prod_{i=1}^{4} \bar{z}_{i}, \prod_{i=5}^{8} \bar{z}_{i}, \ldots, \prod_{i=4 t-3}^{4 t} \bar{z}_{i}\right)
$$

From the equations (1) it can be verified that (2) is satisfied.

Warm-up for the RANDOM gadget. The second and last gadget that we need is called RANDOM and is essentially applied to every bundle in $\widehat{C}$ that corresponds to a wire in $C$. This gadget has to satisfy two properties. First we need that on input $x \in G^{t}$, the RANDOM gadget outputs a bundle $z \in G^{t}$ that is distributed uniformly over $\left\{z \in G^{t} \mid \prod_{i} z_{i}=\prod_{i} x_{i}\right\}$. This is necessary both for the correctness and security of the construction. The second property, necessary only for the security, is that given an input-output pair $(x, z)$ for this gadget, we should be able to compute locally a distribution on the gadget's wires that is indistinguishable from the real distribution. (This allows us to replace the real distribution on the wires of $\widehat{C}$ with the one in which each RANDOM gadget is reconstructed. Then we can replace each bundle of wires in $\widehat{C}$ with a uniform bundle, which the simulator can do by itself, and blame any inconsistency on the reconstructor.) This property is called local reconstructibility and is a variant of the one in $\left[\mathrm{FRR}^{+} 10\right]$.

Before describing our gadget, we note that there is a simple gadget that satisfies the first property but not the second. Namely, choose $r_{1}, \ldots, r_{t-1} \in G$ uniformly at random, and output

$$
z=\left(x_{1} \cdot r_{1}, r_{1}^{-1} \cdot x_{2} \cdot r_{2}, \ldots, r_{t-1}^{-1} \cdot x_{t}\right) .
$$

Indeed, this basic re-randomization technique has been used to great effect in a number of works, e.g. [Kil88, ?, AIK06, $\mathrm{GGH}^{+} 08$, AAW10]. However, this simple gadget does not satisfy local reconstructibility. One reason is that given $x, z$, one can come up with values for the $r_{i}$ that are consistent with each gate in the circuit if and only if $\prod x_{i}=\prod z_{i}$. However, the latter is an $\mathrm{NC}^{1}$-hard question, whereas consistency of the $r$ may be checked by, say, a DNF.

By contrast, one feature of our gadget is that given $x, z$ one can produce consistent values for the wires even if $\prod x_{i} \neq \prod z_{i}$. The catch is that in the latter case the values of certain constant gates are not chosen as in the correct implementation, but the leakage functions will not be able to distinguish this.

The RANDOM gadget. We now describe our gadget. The computation corresponds to replacing each pair $\left(r_{i}, r_{i}^{-1}\right)$ in (3) with a pair $(R, L) \in G^{t} \times G^{t}$ such that $\left(\prod_{j} R_{j}\right) \cdot\left(\prod_{j} L_{j}\right)=$ id, and then computing the multiplications in a specific order.

First, choose $R^{(1)}, \ldots, R^{(t-1)} \in G^{t}$ uniformly at random. Next, choose $L^{(2)}, \ldots, L^{(t)} \in G^{t}$ at random conditioned on

$$
\prod_{j} L_{j}^{(i)}=\left(\prod_{j} R_{j}^{(i-1)}\right)^{-1}
$$


for $1<i \leq t$. In the single-query setting, we think of Comp choosing these values and hardwiring them into $\widehat{C}$; in the multi-query setting, each pair $\left(R^{(i-1)}, L^{(i)}\right)$ will be output by a secure hardware component. We will drop the superscripts on $R$ and $L$ when they are clear from context. Condition (4) implies the following equation.

$$
\left(x_{1} \cdot \prod_{j} R_{j}^{(1)}\right) \cdot\left(\prod_{j} L_{j}^{(2)} \cdot x_{2} \cdot \prod_{j} R_{j}^{(2)}\right) \cdots\left(\prod_{j} L_{j}^{(t)} \cdot x_{t}\right)=\prod_{i} x_{i} .
$$

So, we compute $z$ by letting $z_{i} \in G$ be the result of the $i$ th parenthesized expression in (5). Clearly this $z$ has the correct distribution. We perform each iterated multiplication by a depth- $O(t)$ tree of fan-in-2 multiplication gates in a specific way, described now.

For $z_{1}$, the product is computed in the straightforward way from left to right by a depth- $t$ tree that computes each prefix product

$$
\lambda_{m}:=x_{1} \cdot \prod_{j=1}^{m} R_{j}=\lambda_{m-1} \cdot R_{m}
$$

for $m=1, \ldots, t$ in order, and outputs $z_{1}:=\lambda_{t}$. The product for $z_{t}$ is computed in the straightforward way from left to right as well.

Now let $1<i<t$. The product for $z_{i}$ is computed by a depth- $2 t$ tree that multiplies "from the inside out". That is, it computes in order a sequence $\lambda_{1}, \ldots, \lambda_{2 t-1}$ defined by $\lambda_{1}:=L_{t} \cdot x_{i}$ and recursively for $j=1, \ldots, t-1$ by

$$
\begin{aligned}
\lambda_{2 j} & :=\lambda_{2 j-1} \cdot R_{j} \\
\lambda_{2 j+1} & :=L_{t-j} \cdot \lambda_{2 j}
\end{aligned}
$$

and then outputs $z_{i}:=\lambda_{2 t-1} \cdot R_{t}$.

By way of illustration, when $t=3$ the sequence is computed as follows.

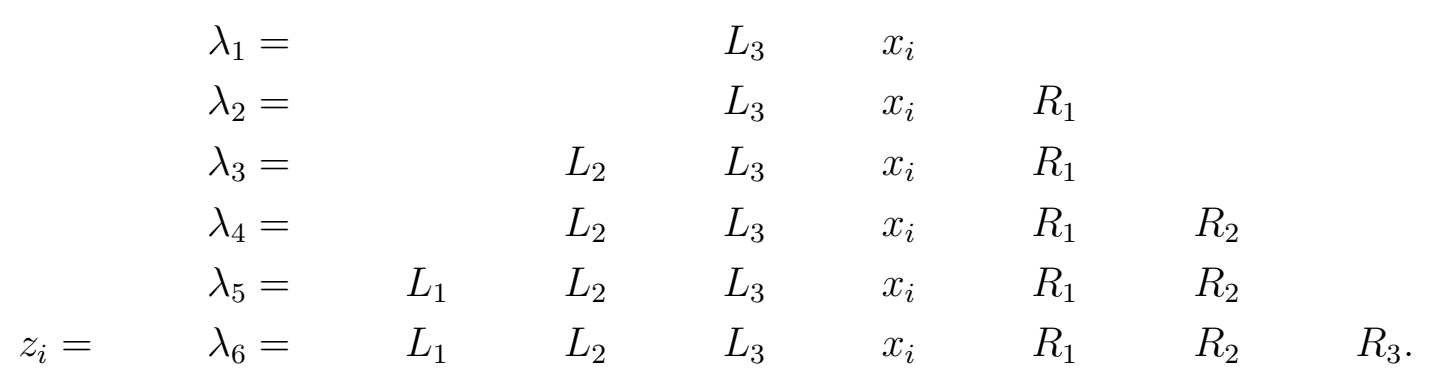

It is interesting to note that that the size overhead of $O\left(t^{2}\right)$ in our construction comes from the fact that the RANDOM gadget has this size, and not from the NAND gadget which has size $O(t)$. This is in contrast to previous constructions using the parity encoding (e.g. [ISW03, $\left.\mathrm{FRR}^{+} 10\right]$ ), for which it is not known how to compute Nand with this size. Improving the $O\left(t^{2}\right)$ overhead in any of these constructions, including ours, is an interesting open problem.

The following key lemma in this work shows that the RANDOM gadget is locally reconstructible. We say that $x, z \in G^{t}$ are plausible if it is $\operatorname{possible~for~} \operatorname{RANDOM}(x)$ to output $z$, i.e. if $\prod_{i} x_{i}=\prod_{i} z_{i}$. 
Lemma 2.1. There is a poly $(t)$-time computable distribution on 1-local functions $\mathrm{R}_{\mathrm{RANDOM}}$ : $G^{t} \times G^{t} \rightarrow G^{\mid \text {RANDOM| }}$ for which the following holds. Let $W_{x \rightarrow z}$ denote the distribution on the wires of $z=\operatorname{RANDOM}(x)$. For any $\ell$ with domain $G^{\text {|RAnDom| }}$ and any plausible $x, z \in G^{t}$, if

$$
\Delta\left(\ell\left(\mathrm{R}_{\mathrm{RANDOM}}(x, z)\right), \ell\left(W_{x \rightarrow z}\right)\right)>\epsilon \cdot(t-1)
$$

then some 1-local extension of $\ell$ is not $\epsilon$-fooled by $G$, i.e., there is a $g \in G$ and a 1-local function $f: G^{t} \rightarrow G^{\mid \text {RANDOM| }}$ such that

$$
\Delta\left(\ell\left(f\left(D_{g}\right)\right), \ell\left(f\left(U_{G^{t}}\right)\right)\right)>\epsilon .
$$

Proof. We first describe an alternate procedure for generating $W_{x \rightarrow z}$. Fix any plausible $x, z \in G^{t}$. For the tree computing $z_{1}$, choose each $\lambda_{j}$ uniformly at random for $j=1, \ldots, t-1$, and compute each $R_{j}^{(1)}:=\lambda_{j-1}^{-1} \cdot \lambda_{j}$ for $j=1, \ldots, t$, defining $\lambda_{t}:=z_{1}$ and $\lambda_{0}:=x_{1}$. Choose the wires for the tree computing $z_{t}$ analogously. Then for $i=2, \ldots, t-1$ in order, choose the wires for the tree computing $z_{i}$ as follows.

1. Choose $L^{(i)} \in G^{t}$ uniformly over vectors with product $=\left(\prod_{j} R_{j}^{(i-1)}\right)^{-1}$.

2. For $j=1, \ldots, t-1$ choose $\lambda_{2 j} \in G$ uniformly at random.

3. For $j=0, \ldots t-1$ compute $\lambda_{2 j+1}:=L_{t-j}^{(i)} \cdot \lambda_{2 j}$, where $\lambda_{0}:=x_{i}$.

4. For $j=1, \ldots, t$ compute $R_{j}^{(i)}:=\lambda_{2 j-1}^{-1} \cdot \lambda_{2 j}$, where $\lambda_{2 t}:=z_{i}$.

To show that this distribution is identical to $W_{x \rightarrow z}$, it is enough to observe that the vectors $R^{(i)}, L^{(i)}$ are distributed correctly, i.e. uniformly conditioned on (4) and on the $i$ th parenthesized expression in (5) equalling $z_{i}$ for all $i$. This is because the above procedure computes consistent wire values, and the $R^{(i)}, L^{(i)}$ (along with $x, z$ ) determine the values of all other wires. $R^{(1)}$ and $L^{(t)}$ are clearly distributed correctly. Then for each $1<i<t, L^{(i)}$ is distributed correctly assuming that $R^{(i-1)}$ is, $R_{j}^{(i)}$ is uniform for $1 \leq j<t$, and $R_{t}^{(i)}$ takes the unique consistent value.

This computation is sequential, due to the selection of $L^{(i)}$ based on $R^{(i-1)}$ in step 1 . This selection is there to ensure condition (4). However by dropping this condition, we can break the dependencies between multiplication trees and give a local reconstructor. Namely, we define $\mathrm{R}_{\text {RANDOM }}(x, z)$ to be the above computation except that $L^{(i)}$ is chosen uniformly at random in step 1 . Note that $R_{\text {RANDOM }}$ is a distribution on 1-local functions.

To prove the lemma, we define a set of hybrid distributions $H_{m}$ on the wires of RANDOM for $m=1, \ldots, t-1$. Fix any plausible $x, z \in G^{t}$. In $H_{m}$, the wires in the tree computing $z_{i}$ for $i \leq m$ are chosen as in $W_{x \rightarrow z}$, and for $i>m$ the wires are chosen as in $\mathrm{R}_{\mathrm{RANDOM}}(x, z)$. Then, we have $H_{1} \equiv \mathrm{R}_{\mathrm{RANDOM}}(x, z)$ and $H_{t-1} \equiv W_{x \rightarrow z}$ (note that the first and last trees are distributed identically in $W_{x \rightarrow z}$ and $\mathrm{R}_{\text {RAnDom }}(x, z)$ ). Thus if there is a function $\ell$ such that

$$
\Delta\left(\ell\left(\mathrm{R}_{\mathrm{RANDOM}}(x, z)\right), \ell\left(W_{x \rightarrow z}\right)\right)>\epsilon \cdot(t-1)
$$

then there is an $m \in[2, t-1]$ such that

$$
\Delta\left(\ell\left(H_{m-1}\right), \ell\left(H_{m}\right)\right)>\epsilon .
$$


Now let $g \in G$ be the fixed value (depending on $x, z)$ such that $\prod_{j} R_{j}^{(m-1)}=g$ with probability 1 in both $H_{m}$ and $H_{m-1}$. Thus in $H_{m-1}$ (resp. $\left.H_{m}\right), L^{(m)}$ is distributed according to $U_{G^{t}}$ (resp. $D_{g^{-1}}$ ). (Note that this $g$ is arbitrary, i.e. not only $\alpha$ or id, and hence we are using the full generality of Definition 1.2.) Because $H_{m-1}$ and $H_{m}$ differ only in the tree computing $z_{m}$, and because the distribution on these wires is independent of all other wires when $x$ and $z$ are fixed, by an averaging argument we can fix all wires outside this tree while preserving (6). Then given a vector $v \in G^{t}$ distributed according to either $U_{G^{t}}$ or $D_{g^{-1}}$, a 1-local function (of $v$ ) can generate $H_{m-1}$ or $H_{m}$ by setting $L^{(m)}:=v$ and performing steps 2-4 in the above computation. Fixing the randomness of this function by an averaging argument, we obtain the function $f: G^{t} \rightarrow G^{\text {RANDOM } \mid}$ in the statement of the lemma.

Using the above gadgets and Lemma 2.1 one may complete the proof of Theorem 1.4 essentially following $\left[\mathrm{FRR}^{+} 10\right.$, Lemma 13]. For completeness we include a proof in $\S 4$.

\section{On compressing group products}

In this section we show that $\left(A_{5}\right)^{t}$ fools in the sense of Definition 1.2 a number of computational models, proving Theorem 1.5. As mentioned in $\S 1.1$, the results of this section can be viewed as giving compression bounds against a variety of classes for the task of computing $A_{5}$-products. We start by recalling a number of facts related to groups and computation. Then in each of the subsections we analyze each computational model in turn.

First, it will be convenient later to prove that $\ell\left(D_{\alpha}\right)$ and $\ell\left(D_{\text {id }}\right)$ are close for every $\alpha \in G$, $\ell \in \mathcal{L}$, and we observe that this is sufficient.

Lemma 3.1. If $\Delta\left(\ell\left(D_{\alpha}\right), \ell\left(D_{\mathrm{id}}\right)\right) \leq \epsilon$ for every $\alpha \in G$ and every $\ell$ in the 1-local extension of $\mathcal{L}$, then $\mathcal{L}$ is $\epsilon$-fooled by $G^{t}$.

Proof. If $\mathcal{L}$ is not $\epsilon$-fooled by $G^{t}$ then $\exists \alpha \in G, \ell \in \mathcal{L}$ such that $\Delta\left(\ell\left(D_{\alpha}\right), \ell\left(U_{G^{t}}\right)\right)>\epsilon$. Then by an averaging argument, $\exists \beta \in G$ such that $\Delta\left(\ell\left(D_{\alpha}\right), \ell\left(D_{\beta}\right)\right)>\epsilon$. Defining $\ell^{\prime}\left(x_{1}, \ldots, x_{t}\right):=$ $\ell\left(x_{1}, \ldots, x_{t} \cdot \beta\right)$ in the 1 -local extension of $\mathcal{L}$, we have $\Delta\left(\ell^{\prime}\left(D_{\alpha \beta^{-1}}\right), \ell^{\prime}\left(D_{\text {id }}\right)\right)>\epsilon$.

We will also make use of the random self-reducibility of the distributions $D_{\alpha}$.

Lemma 3.2 ([Kil88]). There exists a distribution on 1-local functions $R: G^{t} \rightarrow G^{t}$ such that for any $\alpha \in G$ and any $x$ in the support of $D_{\alpha}, R(x) \equiv D_{\alpha}$.

Proof. $R$ chooses $r_{1}, \ldots, r_{t-1} \in G$ uniformly and outputs $\left(x_{1} r_{1}, r_{1}^{-1} x_{2} r_{2}, \ldots, r_{t-1}^{-1} x_{t}\right)$.

Recall the following standard terminology: $\alpha$ is an involution if $\alpha=\alpha^{-1}$, and $\alpha$ is the commutator of $\beta$ and $\gamma$ if $\alpha=\beta \gamma \beta^{-1} \gamma^{-1}$. We say that $M:\{0,1\}^{n} \rightarrow\left(A_{5}\right)^{*} \alpha$-computes a function $f:\{0,1\}^{n} \rightarrow\{0,1\}$ if for every $x, \prod_{i} M(x)_{i}=\alpha^{f(x)}\left(\right.$ where $\alpha^{0}=$ id and $\alpha^{1}=\alpha$ ).

The next theorem follows from [Bar89, Theorem 5] because every element of $A_{5}$ is a commutator.

Theorem 3.3 ([Bar89]). For every $\alpha \in A_{5}$ and every fan-in-2 circuit $C:\{0,1\}^{n} \rightarrow\{0,1\}$ of depth $d$, there is a 1-local function $M:\{0,1\}^{n} \rightarrow\left(A_{5}\right)^{O\left(4^{d}\right)}$ that $\alpha$-computes $C$. 
Moreover, let $f_{1}, \ldots, f_{m}:\{0,1\}^{n} \rightarrow\{0,1\}$ be functions such that for each $i \leq m$ there is a 1-local function $M_{i}^{\prime}:\{0,1\}^{n} \rightarrow\left(A_{5}\right)^{n}$ that $\alpha$-computes $f_{i}$. Then, for every fan-in-2 circuit $C:\{0,1\}^{m} \rightarrow\{0,1\}$ of depth $d$ there is a 1-local function $M:\{0,1\}^{m n} \rightarrow\left(A_{5}\right)^{O\left(n \cdot 4^{d}\right)}$ that $\alpha$-computes $C\left(f_{1}(\cdot), \ldots, f_{m}(\cdot)\right)$.

The following two lemmas allow certain functions to be more efficiently $\alpha$-computed. These can be compared with the works by Cai and Lipton [CL94] and Cleve [Cle91] which give increasingly efficient versions of Barrington's construction (here efficiency is measured in the length of M's output). Our construction is simpler than the ones given in these works, but also less general.

Lemma 3.4. For every involution $\alpha \in A_{5}$, the following holds.

1. There is a 1-local function $M:\{0,1\}^{n} \rightarrow\left(A_{5}\right)^{n}$ that $\alpha$-computes $\bigoplus_{i=1}^{n} x_{i}$.

2. If $M$ and $M^{\prime} \alpha$-compute some Boolean functions $f$ and $f^{\prime}$, then their concatenation $\left(M, M^{\prime}\right) \alpha$-computes the function $f \oplus f^{\prime}$.

Proof. For the first item: given input $x \in\{0,1\}^{n}$, output $y \in\left(A_{5}\right)^{n}$ such that $y_{i}=\alpha^{x_{i}}$. The correctness of this construction, as well as the second item, follows from the isomorphism between the group $\{0,1\}$ (under $\oplus$ ) and the subgroup $\{$ id, $\alpha\} \subset A_{5}$.

For the next lemma, note that because every $k$-cycle $\left(\begin{array}{llll}a_{1} & a_{2} & \cdots & a_{k}\end{array}\right)$ can be written as a product of $k-1$ transpositions $\left(a_{1} a_{2}\right)\left(\begin{array}{ll}a_{1} & a_{3}\end{array}\right) \cdots\left(\begin{array}{ll}a_{1} & a_{k}\end{array}\right)$, every element of $A_{5}$ is either a 3-cycle, a 5-cycle, the product of two disjoint transpositions, or the identity.

Lemma 3.5. Every non-involution in $A_{5}$ is the commutator of two involutions.

Proof. Let $a, b, c, d, e$ denote arbitrary, distinct elements of $\{1, \ldots, 5\}$. Every non-involution in $A_{5}$ is either a 3-cycle $(a b c)$ which is the commutator of involutions $(a b)(d e)$ and $(b c)(d e)$, or a 5-cycle $(a b c d e)$ which is the commutator of involutions $(b e)(c d)$ and $(a d)(b c)$.

\subsection{Multi-party protocols}

In this section we consider functions computable by a multi-party communication protocol in the "number on forehead" model [CFL83], defined as follows. A protocol $P$ with $n$-bit inputs consists of $k=k(n)$ parties, each with unlimited computational power. The input $x \in\{0,1\}^{k n}$ is partitioned into $k$ blocks, and party $i$ sees all input bits except those in the $i$ th block. The parties communicate in the broadcast model, so every bit sent is seen by all parties. The $(m=m(n))$-bit output of $P$ is defined to be the final $m$ bits that are broadcast, and the cost of $P$ is the total number of bits broadcast by all parties. When $P$ 's input comes from a group $G$, we assume some canonical representation of $G$ 's elements as $(\log |G|)$-bit strings.

We prove the following compression bound for such protocols.

Theorem 3.6. There is a partition of the inputs in $\left(A_{5}\right)^{t}$ into $k$ pieces such that any $k$-party protocol communicating $c$ bits and outputting $\leq c$ bits is $\epsilon$-fooled by $\left(A_{5}\right)^{t}$ for $\epsilon=2^{c-\Omega\left(t /\left(k^{2} 4^{k}\right)\right)}$. 
We prove this theorem by combining an efficient translation from bits to group products with the following lower bound. Define the generalized inner-product function $G I P_{n, k}$ : $\{0,1\}^{n k} \rightarrow\{0,1\}$ as

$$
G I P_{n, k}(x):=\bigoplus_{i=1}^{n} \bigwedge_{j=1}^{k} x_{i, j} .
$$

Then we have the following lemma, originally due to Babai, Nisan, and Szegedy [BNS92] and with increasingly streamlined proofs in [CT93, Raz00, VW08].

Lemma 3.7 ([BNS92]). There is a partition of the inputs to $G I P_{n, k}$ into $k$ blocks such that for every protocol $P:\{0,1\}^{n k} \rightarrow\{0,1\}$ with $k$ parties that communicates at most $c$ bits, $\operatorname{Pr}_{x}\left[P(x)=G I P_{n, k}(x)\right] \leq \frac{1}{2}+2^{c-\Omega\left(n / 4^{k}\right)}$.

We give the following translation to bits from group products.

Lemma 3.8. For every $\alpha \in A_{5}$, there is a 1-local function $M:\{0,1\}^{n k} \rightarrow\left(A_{5}\right)^{O\left(n k^{2}\right)}$ that $\alpha$-computes $G I P_{n, k}$.

Proof. Assume $\alpha$ is an involution. Let $M^{\prime}:\{0,1\}^{k} \rightarrow\left(A_{5}\right)^{O\left(k^{2}\right)}$ be the function guaranteed by Theorem 3.3 that $\alpha$-computes the $k$-wise AND of its input. Then letting $x^{(i)}:=\left(x_{i, 1}, \ldots, x_{i, k}\right)$ for each $i \leq n$, the function

$$
M(x):=\left(M^{\prime}\left(x^{(1)}\right), \ldots, M^{\prime}\left(x^{(n)}\right)\right)
$$

$\alpha$-computes $G I P_{n, k}$ by the second item of Lemma 3.4.

If $\alpha$ is not an involution, let $\beta, \gamma \in A_{5}$ be the involutions guaranteed by Lemma 3.5 such that $\alpha=\beta \gamma \beta \gamma$ (note that $\beta=\beta^{-1}$ and $\gamma=\gamma^{-1}$ ). Then let $M^{\prime}$ instead $\beta$-compute the AND of its input, and compute $M$ as

$$
M(x):=\left(M^{\prime}\left(x^{(1)}\right), \ldots, M^{\prime}\left(x^{(n)}\right), \gamma, M^{\prime}\left(x^{(1)}\right), \ldots, M^{\prime}\left(x^{(n)}\right), \gamma\right) .
$$

We now give the proof of Theorem 3.6.

Proof of Theorem 3.6. For an appropriate $n=\Omega\left(t / k^{2}\right)$, let $M:\{0,1\}^{n \cdot k} \rightarrow\left(A_{5}\right)^{t}$ be the 1 -local function guaranteed by Lemma 3.8 that $\alpha$-computes $G I P_{n, k}$. Consider the partition on the $t$ elements of the input from $\left(A_{5}\right)^{t}$ that is induced by $M$ from the partition on $G I P_{n, k}$ guaranteed by Lemma 3.7.

Assume for contradiction that some protocol on this partition is not $\epsilon$-fooled. Without loss of generality the protocol outputs 1 bit. (The last player can simulate whatever set maximizes the statistical distance of the multi-bit protocol output distributions.) By Lemma 3.1, there is an $\alpha \in A_{5}$ and a protocol $P:\left(A_{5}\right)^{t} \rightarrow\{0,1\}$ with $k$ parties communicating $\leq c$ bits such that $\Delta\left(P\left(D_{\alpha}\right), P\left(D_{\text {id }}\right)\right) \geq 2^{c-\beta t /\left(k^{2} 4^{k}\right)}$, for a suitable constant $\beta$.

By combining the $P$ with $M$ we now give a distribution on protocols $P^{\prime}:\{0,1\}^{n \cdot k} \rightarrow$ $\{0,1\}$ for $G I P_{n, k}$ with the same number of parties, the same communication, and the same advantage up to the constant $\beta$. This contradicts Lemma 3.7.

On input $x$, each party in $P^{\prime}$ first computes the portion of $y:=M(x) \in\left(A_{5}\right)^{t}$ that depends on the input bits it can see; this is done with no communication as $M$ is 1-local. 
Next each party computes the portion of $z:=\left(y_{1} \cdot r_{1}, r_{1}^{-1} \cdot y_{2} \cdot r_{2}, \ldots, r_{t-1}^{-1} \cdot y_{t}\right)$ that depends on the input bits it can see, again with no communication. ( $r$ is a public random string.) Finally the parties compute and output $P(z)$ using the protocol $P$.

Note for every $x$ such that $G I P_{n, k}(x)=1$ (resp. $G I P_{n, k}(x)=0$ ), $z$ is distributed according to $D_{\alpha}$ (resp. $D_{\text {id }}$ ) over the choice of $r$. The proof is now completed using the fact that $\operatorname{Pr}_{x}\left[G I P_{n, k}(x)=0\right]>\operatorname{Pr}_{x}\left[G I P_{n, k}(x)=1\right] \geq 1 / 2-2^{-\Omega\left(n / 2^{k}\right)}$ [VW08, Claim 2.11].

\section{$3.2 \mathrm{TC}^{0}$}

In this section we observe that, because computing products over $A_{5}$ is complete for $\mathrm{NC}^{1}$, if $\mathrm{TC}^{0} \neq \mathrm{NC}^{1}$ then the set of $\mathrm{TC}^{0}$ circuits with $O(\log t)$ bits of output is $t^{-\omega(1)}$-fooled by $\left(A_{5}\right)^{t}$.

Recall that $\mathrm{NC}^{1}$ is the class of poly-size fan-in-2 And/Or/Not circuits with depth $O(\log n)$, and $\mathrm{TC}^{0}$ is the class of poly-size unbounded-fan-in constant-depth circuits where each gate computes, for some $c$, the $c$-threshold function which is 1 iff $\geq c$ inputs are 1 .

The high-level idea behind the next theorem is the following. Assume there is an $\alpha \in A_{5}$ and a $\mathrm{TC}^{0}$ circuit $C$ that can distinguish between $D_{\alpha}$ and $D_{\text {id }}$ with advantage $\geq t^{-k}$ for some $k$. Then for any $\mathrm{NC}^{1}$ circuit $B$ we construct a $\mathrm{TC}^{0}$ circuit that, on input $x$, chooses $m=t^{O(k)}$ samples from the distribution $D_{\alpha^{B(x)}}$ and outputs 1 iff the number of samples on which $C$ outputs 1 is sufficiently close to $m \cdot \operatorname{Pr}\left[C\left(D_{\alpha}\right)=1\right]$. This last check can be computed with threshold gates, and we can sample from $D_{\alpha^{B(x)}}$ by using Theorem 3.3 to obtain a single element in its support and then relying on the random self-reducibility of this distribution.

Theorem 3.9. If $\mathrm{TC}^{0} \neq \mathrm{NC}^{1}$ then $\forall k$ and infinitely many $t$, the class $\mathcal{L}$ of $\mathrm{TC}^{0}$ circuits with size $\leq t^{k}$ and output length $k \log t$ is $t^{-k}$-fooled by $\left(A_{5}\right)^{t}$.

Proof. Assume that $\exists k$ such that for sufficiently large $t, \mathcal{L}$ is not $t^{-k}$-fooled by $\left(A_{5}\right)^{t}$. Then by Lemma 3.1 there exists an $\alpha \in A_{5}$ and a $\mathrm{TC}^{0}$ circuit $C:\left(A_{5}\right)^{t} \rightarrow\{0,1\}^{k \log t}$ with size $\leq t^{k}$ such that $\Delta\left(C\left(D_{\alpha}\right), C\left(D_{\mathrm{id}}\right)\right) \geq t^{-k}$. Let $S \subseteq\{0,1\}^{k \log t}$ be the set that maximizes $\operatorname{Pr}\left[C\left(D_{\alpha}\right) \in S\right]-\operatorname{Pr}\left[C\left(D_{\text {id }}\right) \in S\right]$, and note that checking $x \in S$ can be done by a TC ${ }^{0}$ circuit of size $t^{O(k)}$. Thus, there is a $\mathrm{TC}^{0}$ circuit $C^{\prime}:\left(A_{5}\right)^{t} \rightarrow\{0,1\}$ of size $t^{O(k)}$ such that

$$
\operatorname{Pr}\left[C^{\prime}\left(D_{\alpha}\right)=1\right]-\operatorname{Pr}\left[C^{\prime}\left(D_{\mathrm{id}}\right)=1\right] \geq t^{-k}
$$

Define $\epsilon_{\alpha}:=\operatorname{Pr}\left[C^{\prime}\left(D_{\alpha}\right)=1\right]$ and $\epsilon_{\text {id }}:=\operatorname{Pr}\left[C^{\prime}\left(D_{\text {id }}\right)=1\right]$, and note that $\epsilon_{\alpha} \geq t^{-k}$.

Let $B:\{0,1\}^{n} \rightarrow\{0,1\}$ be any $\mathrm{NC}^{1}$ circuit, and for an appropriate $t=n^{O(1)}$ let $M:\{0,1\}^{n} \rightarrow\left(A_{5}\right)^{t}$ be the 1-local function (guaranteed by Theorem 3.3) that $\alpha$-computes $B$. Let $C^{\prime \prime}:\{0,1\}^{n} \rightarrow\{0,1\}$ be the randomized $\mathrm{TC}^{0}$ circuit that performs the following steps on input $x \in\{0,1\}^{n}$.

1. Compute $y=M(x) \in\left(A_{5}\right)^{t}$.

2. For $m:=t^{3 k}(n+2) / \epsilon_{\alpha}=n^{O(k)}$, sample $z_{1}, \ldots, z_{m} \in\left(A_{5}\right)^{t}$ independently from $D_{\alpha^{B(x)}}$ by computing $R(y)$ where $R$ is the 1-local function from Lemma 3.2.

3. Use two layers of threshold gates to output 1 iff

$$
\left(1-1 /\left(2 t^{k}\right)\right) \cdot m \epsilon_{\alpha} \leq \sum_{i=1}^{m} C^{\prime}\left(z_{i}\right) \leq\left(1+1 /\left(2 t^{k}\right)\right) \cdot m \epsilon_{\alpha} .
$$


We now prove the following claim.

Claim. $\forall x \in\{0,1\}^{n}: \operatorname{Pr}\left[C^{\prime \prime}(x)=B(x)\right] \geq 1-2^{-n-1}$ over the random coins of $C^{\prime \prime}$.

This implies the theorem, as follows. By a union bound there is a way to fix the random coins of $C^{\prime \prime}$ such that $C^{\prime \prime}(x)=B(x)$ for every $x$. Then because $B \in \mathrm{NC}^{1}$ was arbitrary and $C^{\prime \prime} \in \mathrm{TC}^{0}$, we have $\mathrm{TC}^{0}=\mathrm{NC}^{1}$.

Proof of Claim. Denote $X:=\sum_{i=1}^{m} C^{\prime}\left(z_{i}\right)$, and $\mu:=\mathbb{E}[X]$.

Fix $x$, and first assume $B(x)=1$ which means $\mu=m \epsilon_{\alpha}=t^{3 k}(n+2)$. Then

$$
\operatorname{Pr}\left[C^{\prime \prime}(x)=B(x)\right]=\operatorname{Pr}\left[|X-\mu| \leq \mu /\left(2 t^{k}\right)\right] \geq 1-2 e^{-\mu \cdot t^{-3 k}} \geq 1-2^{-n-1}
$$

by a Chernoff bound.

Now assume $B(x)=0$. Then $\mu=m \epsilon_{\text {id }}$, and since $\epsilon_{\alpha} / \epsilon_{\text {id }} \geq 1+1 / t^{k}$ by $(7)$, we have $\left(1-1 /\left(2 t^{k}\right)\right) \cdot m \epsilon_{\alpha} \geq \mu\left(1+1 /\left(3 t^{k}\right)\right)$. Then using another Chernoff bound, we have

$$
\operatorname{Pr}\left[C^{\prime \prime}(x)=B(x)\right] \geq 1-\operatorname{Pr}\left[X \geq \mu\left(1+1 /\left(3 t^{k}\right)\right)\right] \geq 1-e^{-\mu \cdot t^{-3 k}} \geq 1-2^{-n-1} .
$$

This completes the proof of the theorem.

\section{$3.3 \quad \mathrm{AC}^{0}$}

In this section, we observe that by combining the following compression bound against $\mathrm{AC}^{0}$ due to Dubrov and Ishai [DI06] with our Lemma 3.4 above, we can obtain a quantitatively identical compression bound for $A_{5}$-products against $\mathrm{AC}^{0}$.

Let $\oplus^{-1}(b)$ denote the uniform distribution over $n$-bit strings with parity $=b$.

Theorem 3.10 ([DI06]). For every $0<\delta<1$ and every $d \in \mathbb{N}$, there is a constant $\epsilon>0$ such that the following holds. For every unbounded-fan-in circuit $C:\{0,1\}^{n} \rightarrow\{0,1\}^{n^{\delta}}$ of depth $\leq d$ and size $\leq 2^{\epsilon \cdot n^{(1-\delta) / d}}$ :

$$
\Delta\left(C\left(\oplus^{-1}(0)\right), C\left(\oplus^{-1}(1)\right)\right)<2^{-\epsilon \cdot n^{(1-\delta) / d}} .
$$

Theorem 3.11. For every $0<\delta<1$ and every $d \in \mathbb{N}$, there is a constant $\epsilon>0$ such that the following holds. Let $\mathcal{L}$ be the class of unbounded-fan-in circuits $C:\left(A_{5}\right)^{t} \rightarrow\{0,1\}^{t^{\delta}}$ of depth $\leq d$ and size $\leq 2^{\epsilon \cdot t^{(1-\delta) / d}}$. Then, $\mathcal{L}$ is $2^{-\epsilon \cdot t^{(1-\delta) / d}}$-fooled by $\left(A_{5}\right)^{t}$.

Proof. Assume for contradiction that $\mathcal{L}$ is not $2^{-\epsilon \cdot t^{(1-\delta) / d}}$-fooled by $\left(A_{5}\right)^{t}$. Then by Lemma 3.1 there is a circuit $C \in \mathcal{L}$ and an $\alpha \in A_{5}$ such that

$$
\Delta\left(C\left(D_{\alpha}\right), C\left(D_{\text {id }}\right)\right) \geq 2^{-\epsilon \cdot t^{(1-\delta) / d}} .
$$

For $n=\Omega(t)$, let $M:\{0,1\}^{n} \rightarrow\left(A_{5}\right)^{t}$ be the 1-local function guaranteed by Lemmas 3.4 and 3.5 such that

$$
\prod_{i=1}^{t} M(x)_{i}= \begin{cases}\text { id } & \text { if } \oplus_{j=1}^{n} x_{j}=0 \\ \alpha & \text { if } \oplus_{j=1}^{n} x_{j}=1 .\end{cases}
$$


Let $R$ be the function from Lemma 3.2. Then we have

$$
\Delta\left(C\left(R\left(M\left(\oplus^{-1}(0)\right)\right)\right), C\left(R\left(M\left(\oplus^{-1}(1)\right)\right)\right)\right) \geq 2^{-\epsilon \cdot t^{(1-\delta) / d}}=2^{-\epsilon^{\prime} \cdot n^{\left(1-\delta^{\prime}\right) /(d+1)}}
$$

for appropriately chosen $\epsilon^{\prime}=\epsilon^{\prime}(\epsilon)$ and $\delta^{\prime}=\delta^{\prime}(\delta)$. Noting that the depth of $C(R(M(\cdot)))$ is $d+1$ and using an averaging argument to fix the randomness of $R$, this contradicts Theorem 3.10 .

\section{4 $\mathrm{AC}^{0}$ with symmetric gates}

Here we show that our hardness assumption holds when $\mathcal{L}$ is the class of unbounded-fan-in constant-depth circuits that contain $t^{O(\log t)}$ And/Or/Not gates and $O\left(\log ^{2} t\right)$ gates that each compute an arbitrary symmetric function. Specifically, we prove the following.

Theorem 3.12. For every $d$, there is an $\epsilon>0$ such that the following holds for every $t$.

Let $\mathcal{L}$ be the set of functions $\ell:\left(A_{5}\right)^{t} \rightarrow\{0,1\}^{t^{0.1}}$ where each output bit of $\ell$ is computable by an unbounded-fan-in circuit of depth $\leq d$ that contains $\leq t^{\epsilon \log t}$ And/Or/Not gates and $\leq \epsilon \log ^{2} t$ arbitrary symmetric gates.

Then, $\mathcal{L}$ is $t^{-\epsilon \log t}$-fooled by $\left(A_{5}\right)^{t}$.

Note that in fact these circuits have up to $O\left(t^{0.1} \cdot \log ^{2} t\right)$ arbitrary symmetric gates, though each output bit only depends on $O\left(\log ^{2} t\right)$ of them.

The proof of Theorem 3.12 extends a lower bound due to Viola [Vio07] and combines it with an efficient translation from bits to group products. Viola's lower bound shows that the following function $P A P_{n, m}:\{0,1\}^{n^{2} m} \rightarrow\{0,1\}$ (for "parity-and-parity") is hard on average for this circuit class to compute.

$$
P A P_{n, m}(x):=\bigoplus_{i=1}^{n} \bigwedge_{j=1}^{m} \bigoplus_{k=1}^{n} x_{i, j, k}
$$

We use the following translation from PAP-inputs to group products.

Theorem 3.13. For every $\alpha \in A_{5}$, there is a 1-local function $M:\{0,1\}^{n^{2} m} \rightarrow\left(A_{5}\right)^{O\left(n^{2} m^{2}\right)}$ that $\alpha$-computes $P A P_{n, m}$.

The proof of this theorem is analogous to that of Lemma 3.8, using the "moreover" part of Theorem 3.3. We omit the details.

For the remainder of this section, we let $P A P$ denote $P A P_{n, 0.3 \log n}$. By combining Theorem 3.13 with the random self-reducibility of the distributions $D_{\alpha}$, we prove the following.

Lemma 3.14. Let $\mathcal{L}$ be as in Theorem 3.12, and assume that $\mathcal{L}$ is not $t^{-\epsilon \log t}$-fooled by $\left(A_{5}\right)^{t}$. Then there is an $n=\Omega(\sqrt{t} / \log t)$, an $\epsilon^{\prime}=\epsilon^{\prime}(\epsilon)>0$, and a function $\ell^{\prime}:\{0,1\}^{n^{2} \cdot 0.3 \log n} \rightarrow$ $\{0,1\}^{t^{0.1}}$ such that each output bit of $\ell^{\prime}$ is computable by an unbounded-fan-in circuit of depth $\leq d+1$ that contains $\leq n^{\epsilon^{\prime} \log n}$ And/Or/Not gates and $\leq \epsilon^{\prime} \log ^{2} n$ arbitrary symmetric gates, and

$$
\Delta\left(\ell^{\prime}\left(P A P^{-1}(1)\right), \quad \ell^{\prime}\left(P A P^{-1}(0)\right)\right) \geq n^{-\epsilon^{\prime} \log n} .
$$


Proof. If $\mathcal{L}$ is not $t^{-\epsilon \log t}$-fooled by $\left(A_{5}\right)^{t}$, then there is an $\alpha \in A_{5}$ and an $\ell \in \mathcal{L}$ such that $\Delta\left(\ell\left(D_{\alpha}\right), \ell\left(D_{\mathrm{id}}\right)\right) \geq t^{-\epsilon \log t}$. For an appropriate $n=\Omega(\sqrt{t} / \log t)$, let $M:\{0,1\}^{n^{2} \cdot 0.3 \log n} \rightarrow$ $\left(A_{5}\right)^{t}$ be the 1-local function guaranteed by Theorem 3.13 that $\alpha$-computes $P A P$. Let $R$ : $\left(A_{5}\right)^{t} \rightarrow\left(A_{5}\right)^{t}$ be the randomized 1-local function from Lemma 3.2. Then $\ell^{\prime}:=\ell(R(M(\cdot)))$ satisfies the lemma, and by an averaging argument we can fix the randomness of $R$ so that $\ell^{\prime}$ is deterministic.

We now prove Theorem 3.12. The lower bound in [Vio07] shows that, when restricted to one output bit and with domain $\{0,1\}^{n^{2} \cdot 0.3 \log n}$, circuits in $\mathcal{L}$ have correlation $n^{-\Omega(\log n)}$ with $P A P$. This is done by showing that with probability $1-n^{-\Omega(\log n)}$ over a random restriction $\rho$ to the input bits of a circuit $C \in \mathcal{L}$, we have both that $\left.P A P\right|_{\rho}=G I P$ and that $\left.C\right|_{\rho}$ is computable by a $(0.3 \log n)$-party protocol communicating $\log ^{5} n$ bits, which triggers the lower bound of Lemma 3.7.

In addition to the translation to group products above, we extend this argument to $t^{0.1}$ output bits by using a union bound to show that $\rho$ satisfies these properties simultaneously for all output bits, again with probability $1-n^{-\Omega(\log n)}$. The protocol now exchanges $t^{0.1} \cdot \log ^{5} n<$ $n^{0.21}$ bits which is still sufficiently small to use Lemma 3.7.

Proof of Theorem 3.12. Assume that $\mathcal{L}$ is not $t^{-\epsilon \log t}$-fooled by $\left(A_{5}\right)^{t}$, and let $n, \epsilon^{\prime}$, and $\ell^{\prime}=$ $\left(\ell_{1}^{\prime}, \ldots, \ell_{t^{0.1}}^{\prime}\right)$ be given by Lemma 3.14. Let $R$ be the following distribution over restrictions $\rho$ on $n^{2} \cdot 0.3 \log n$ bits that leave $n \cdot 0.3 \log n$ bits unrestricted:

- Choose $\rho^{\prime}$ uniformly over all restrictions that leave $\left(n^{2} \cdot 0.3 \log n\right)^{0.9}$ bits unset.

- If $\left.P A P\right|_{\rho^{\prime}}$ has $\geq 1$ input unrestricted per bottom $\oplus$ gate, then choose $\rho^{\prime \prime}$ uniformly over restrictions to the remaining bits that leave exactly 1 input unrestricted per bottom $\oplus$ gate.

- Else, choose $\rho^{\prime \prime}$ uniformly over all restrictions to the remaining bits that leave exactly $n \cdot 0.3 \log n$ bits unrestricted.

- Output $\rho=\rho^{\prime} \circ \rho^{\prime \prime}$.

Say that $\rho$ is $\operatorname{good}$ if $\left.P A P\right|_{\rho}$ has exactly 1 input unrestricted per bottom $\oplus$ gate and for every $i=1, \ldots, t^{0.1},\left.\ell_{i}^{\prime}\right|_{\rho}$ is computable by a $(0.3 \log n)$-party protocol (under any partitioning of the input) exchanging $\log ^{5} n$ bits of communication. Combining [Vio07, Claim $11 \&$ Lemma 12] with a union bound over all $\ell_{i}^{\prime}$, we obtain

$$
\operatorname{Pr}_{\rho \leftarrow R}[\rho \text { is good }] \geq 1-n^{-\Omega(\log n)} .
$$

Because $\Delta\left(\ell^{\prime}\left(P A P^{-1}(1)\right), \ell^{\prime}\left(P A P^{-1}(0)\right)\right) \geq n^{-\epsilon^{\prime} \log n}$, there is a set $S \subseteq\{0,1\}^{t^{0.1}}$ such that

$$
\operatorname{Pr}_{x}\left[\ell^{\prime}(x) \in S \mid P A P(x)=1\right]-\operatorname{Pr}_{x}\left[\ell^{\prime}(x) \in S \mid P A P(x)=0\right] \geq n^{-\epsilon^{\prime} \log n} .
$$

For any $\rho$ that is good, let $P_{\rho}:\{0,1\}^{n \cdot 0.3 \log n} \rightarrow\{0,1\}$ be the following $(0.3 \log n)$-party protocol exchanging $t^{0.1} \cdot \log ^{5} n+1 \leq n^{0.21}$ bits. On input $y$, the parties first compute each $\left.\ell_{i}^{\prime}\right|_{\rho}(y)$ by communicating $t^{0.1} \cdot \log ^{5} n$ bits, and then output 1 iff $\left.\ell^{\prime}\right|_{\rho}(y) \in S$ using one additional bit of communication. 
For every $\rho$ that is good, $\left.P A P\right|_{\rho}$ is equal (up to complementing some inputs) to the generalized inner-product function GIP from $\S 3.1$. Thus, by Lemma 3.7 we have

$$
\operatorname{Pr}_{y}\left[P_{\rho}(y)=\left.P A P\right|_{\rho}(y)\right]<1 / 2+2^{-n^{\Omega(1)}}
$$

for every good $\rho$.

Now notice that choosing a random $x \in\{0,1\}^{n^{2} \cdot 0.3 \log n}$ can be thought of as first choosing $\rho$ from $R$ and then choosing $y$ uniformly over $\{0,1\}^{n \cdot 0.3 \log n}$. Then letting $E_{b}$ denote the event " $\rho$ is good and $\left.P A P\right|_{\rho}(y)=b "$, we have

$$
\begin{aligned}
& \operatorname{Pr}_{x}\left[\ell^{\prime}(x) \in S \mid P A P(x)=1\right]-\operatorname{Pr}_{x}\left[\ell^{\prime}(x) \in S \mid P A P(x)=0\right] \\
& =\operatorname{Pr}_{\rho, y}\left[\left.\ell^{\prime}\right|_{\rho}(y) \in S|P A P|_{\rho}(y)=1\right]-\operatorname{Pr}_{\rho, y}\left[\left.\ell^{\prime}\right|_{\rho}(y) \in S|P A P|_{\rho}(y)=0\right] \\
& \leq \operatorname{Pr}_{\rho, y}\left[\left.\ell^{\prime}\right|_{\rho}(y) \in S \mid E_{1}\right]-\operatorname{Pr}_{\rho, y}\left[\left.\ell^{\prime}\right|_{\rho}(y) \in S \mid E_{0}\right]+\operatorname{Pr}_{\rho}[\rho \text { is not good }] \\
& =\operatorname{Pr}_{\rho, y}\left[P_{\rho}(y)=1 \mid E_{1}\right]-\operatorname{Pr}_{\rho, y}\left[P_{\rho}(y)=1 \mid E_{0}\right]+\operatorname{Pr}_{\rho}[\rho \text { is not good }] \\
& =\operatorname{Pr}_{\rho, y}\left[P_{\rho}(y)=1 \mid E_{1}\right]+\operatorname{Pr}_{\rho, y}\left[P_{\rho}(y)=0 \mid E_{0}\right]-1+\operatorname{Pr}_{\rho}[\rho \text { is not good }] \\
& <\left(1 / 2+2^{-n^{\Omega(1)}}\right) /\left(1 / 2-2^{-n^{\Omega(1)}}\right)-1+\operatorname{Pr}_{\rho}[\rho \text { is not good }] \\
& =2^{-n^{\Omega(1)}}+\underset{\rho}{\operatorname{Pr}}[\rho \text { is not good }] \\
& \leq n^{-\Omega(\log n)}
\end{aligned}
$$

which contradicts (8) for sufficiently small $\epsilon^{\prime}$. Note that the second inequality follows from (9) because $\left.P A P\right|_{\rho}=G I P$ is balanced up to an additive factor of $2^{-n^{\Omega(1)}}$.

\section{Proofs of main theorems}

In this section we prove Theorem 1.4, Corollary 1.6, and Theorem 1.7.

Theorem 1.4. Let $G$ be a group. For every polynomial-time computable function $t=t(n)$, there is a compiler Comp for which the following holds.

1. For every $C:\{0,1\}^{n} \times\{0,1\}^{n} \rightarrow\{0,1\}^{n}$ and $k \in\{0,1\}^{n}$, Comp $(C, k)$ runs in time poly $(|C|, t)$ and outputs a circuit $\widehat{C}$ of size $O\left(t^{2} \cdot|C|\right)$ and depth $O(t \cdot \operatorname{depth}(C))$.

2. For every set of functions $\mathcal{L}$ and every $\epsilon>0$, if the 4 -local extension of $\mathcal{L}$ is $\epsilon$-fooled by $G^{t}$ then Comp is an $(\mathcal{L}, \epsilon \cdot t \cdot|C|)$-leakage-secure compiler.

Proof. Let $C:\{0,1\}^{n} \times\{0,1\}^{n} \rightarrow\{0,1\}^{n}$ and $k \in\{0,1\}^{n}$ be the input to Comp. As described in $\S 2$, Comp constructs a circuit $\widehat{C}$ by replacing each wire in $C$ with a bundle of $t$ wires, and replacing each gate in $C$ with a set of gadgets. Specifically for each Nand gate in in $C$ with two input wires and $m$ output wires, $\overparen{C}$ contains a NAND gadget followed by $m$ RANDOM gadgets in parallel (each of which takes as input the NAND gadget's output).

In order for $\widehat{C}(x)$ to map $\{0,1\}^{n} \rightarrow\{0,1\}^{n}$, it must encode $x \in\{0,1\}^{n}$ to $x^{\prime} \in\left(G^{t}\right)^{n}$ as a first step, and decode $z^{\prime} \in\left(G^{t}\right)^{n}$ to $z \in\{0,1\}^{n}$ as a final step. This is done in the following 
straightforward way. The input encoder sets each $x_{i}^{\prime}$ to be either (id, . ., id) or (id, . ., id, $\alpha$ ) depending if $x_{i}=0$ or 1 . The output decoder computes each product $\prod_{j=1}^{t}\left(z_{i}^{\prime}\right)_{j}$, and sets $z_{i}=0$ or 1 depending if this product is id or $\alpha$. The decoder may use any correct multiplication tree, i.e. the specific tree used is not relevant to the proof of security.

The size/depth bounds of Comp are immediate. To prove that $\widehat{C}$ is a correct circuit (i.e. that $\widehat{C}(x)=C(x, k)$ for every $x)$, one can apply an inductive argument to show that each bundle at the output of a RANDOM gadget correctly encodes the value of the corresponding wire in $C$, and thus the output decoder indeed produces $C(x, k)$.

In the hybrid arguments below, we will crucially use the fact that each bundle of the secret state and each bundle at the output of a RANDOM gadget is uniform (over the random coins of Comp) subject to correctly encoding the corresponding wire of $C$.

On input $(C, x, \widehat{C}(x))$, the simulator $S$ computes a distribution on the wires of $\widehat{C}$ as follows.

First, $S$ computes the wires for the encoder and decoder honestly. For the encoder this is straightforward. For the decoder, $S$ chooses $n$ vectors $z_{i}^{\prime} \in G^{t}$ which are uniform conditioned on the correct product (determined by $\left.\widehat{C}(x)_{i}\right)$, and then computes the wires for the multiplication trees honestly. These wires are distributed identically to the real distribution on $\widehat{C}(x)$ 's wires and thus will not affect the hybrid arguments that follow, which is why these multiplication trees and the complexity of $S$ for this step are not of interest.

Next, $S$ chooses uniformly at random the values for each wire encoding the secret input $k$, as well as each connecting wire at the output of a RANDOM gate (except those which touch the output decoder and have already been chosen).

Next, for each NAND gadget $S$ computes values for its internal wires and for its output wires by simply evaluating the gadget. (Here we use the fact that the output of one NAND gadget is never the input of another, so all NAND input bundles have already been set.)

Finally, $S$ computes internal wire values for each RANDOM gadget using $\mathrm{R}_{\text {RANDOM }}$.

Now let $\widehat{C} \leftarrow \operatorname{Comp}(C, k)$, and recall that $\widehat{W}_{x}$ denotes the real distribution on the wires of $\widehat{C}(x)$. We define an intermediate distribution $W_{x}^{\prime}$ as follows: first draw a sample from $\widehat{W}_{x}$, and then recompute the internal wires of each RANDOM gadget from its input/output bundles using $\mathrm{R}_{\text {RANDOM }}$.

We now show that $W_{x}^{\prime}$ is indistinguishable (by $\mathcal{L}$ ) from both $\widehat{W}_{x}$ and $S(C, x, \widehat{C}(x))$.

Claim 1. If the 1-local extension of $\mathcal{L}$ is $\epsilon$-fooled by $G^{t}$, then $\forall \ell \in \mathcal{L}$ :

$$
\Delta\left(\ell\left(W_{x}^{\prime}\right), \ell\left(\widehat{W}_{x}\right)\right) \leq \epsilon \cdot|C| \cdot(t-1) .
$$

Proof. Assume that there an $\ell \in \mathcal{L}$ such that $\Delta\left(\ell\left(W_{x}^{\prime}\right), \ell\left(\widehat{W}_{x}\right)\right)>\epsilon \cdot|C| \cdot(t-1)$. Define some fixed ordering on the $\leq|C|$ RANDOM gadgets of $\widehat{C}$. Then by a hybrid argument, there is an $m \leq|C|$ and two distributions $H$ and $H^{\prime}$, defined as follows, for which $\Delta\left(\ell(H), \ell\left(H^{\prime}\right)\right)>$ $\epsilon \cdot(t-1) . H$ is defined by first drawing a sample from $\widehat{W}_{x}$, and then recomputing the internal wires of RANDOM gadgets $1, \ldots, m$ from their input/output bundles; $H^{\prime}$ is the same except only random gadgets $1, \ldots, m-1$ are recomputed.

Now by an averaging argument, we can fix all wires in both $H$ and $H^{\prime}$ except those internal to the $m$ th RANDOM gadget, obtaining a function $\ell^{\prime}$ (with domain $G^{\text {RANDOM| }}$ ) in the 1-local extension of $\mathcal{L}$. Then $\ell^{\prime}$ distinguishes the real wires of the $m$ th RANDOM gadget from 
those computed by $\mathrm{R}_{\text {RANDOM }}$ with advantage $>\epsilon \cdot(t-1)$. In combination with Lemma 2.1, this contradicts the claim's hypothesis.

Claim 2. If the 4-local extension of $\mathcal{L}$ is $\epsilon$-fooled by $G^{t}$, then $\forall \ell \in \mathcal{L}$ :

$$
\Delta\left(\ell(S(C, x, \widehat{C}(x))), \ell\left(W_{x}^{\prime}\right)\right) \leq \epsilon \cdot|C| .
$$

Proof. Assume that there an $\ell \in \mathcal{L}$ such that $\Delta\left(\ell(S(C, x, \widehat{C}(x))), \ell\left(W_{x}^{\prime}\right)\right)>\epsilon \cdot|C|$. Define some fixed ordering on the $\leq|C|$ bundles of $\widehat{C}$ that either encode a bit of the secret input $k$ or are at the output of a RANDOM gadget but do not touch the output decoder. Then by a hybrid argument, there is an $m \leq|C|$ and two distributions $H$ and $H^{\prime}$, defined as follows, for which $\Delta\left(\ell(H), \ell\left(H^{\prime}\right)\right)>\epsilon$. In $H$, bundles $1, \ldots, m$ are uniformly random and bundles $m+1, \ldots,|C|$ are random subject to correctly encoding the value of the corresponding wire in $C$; in $H^{\prime}$ only bundles $1, \ldots, m-1$ are uniformly random. In both, each NAND's internal wires are computed using the gadget itself, and each RANDOM's internal wires are computed using $\mathrm{R}_{\text {RANDOM }}$.

Let $g \in\{$ id, $\alpha\}$ be the value encoded by the $m$ th bundle in $W_{x}^{\prime}$ (determined by $C, k$ and $x$ ). Note that the $m$ th bundle is necessarily the input of a NAND gadget, and is either the output of a RANDOM gadget or a bundle encoding a bit of $k$. By an averaging argument, we can fix all wires in $H$ and $H^{\prime}$ while preserving $\Delta\left(\ell(H), \ell\left(H^{\prime}\right)\right)>\epsilon$, except for the following: the $m$ th bundle, the internal and output wires of the NAND gadget that it touches, the internal wires of the RANDOM gadgets that are adjacent to the output of this NAND gadget, and the internal wires of the RANDOM gadget that outputs the $m$ th bundle (if it exists).

Finally, a 4-local function can compute one of the two distributions from an input $v \in G^{t}$ distributed according to either $U_{G^{t}}$ or $D_{g}$ : it plugs $v$ into the $m$ th bundle and computes the (4-local) NAND gadget and the (1-local) $\mathrm{R}_{\text {RANDOM }}$.

Finally, because $\Delta$ is a metric, these two claims give part 2 of the theorem.

Corollary 1.6. There is a single efficient compiler Comp, outputting a circuit $\widehat{C}$ of size $|\widehat{C}|=O\left(|C|^{3}\right)$, that is an $(\mathcal{L}, \epsilon)$-leakage secure compiler for each of the following.

1. $\mathcal{L}=$ number-in-hand protocols with $s$ parties communicating and outputting $\leq \delta \cdot|\widehat{C}|^{1 / 3}$ bits, for a fixed $\delta>0$ and a fixed partition of $\widehat{C}$ into $s=O(1)$ sets; $\epsilon=2^{-\Omega\left(|\widehat{C}|^{1 / 3}\right)}$.

2. $\mathcal{L}=\mathrm{AC}^{0}$ circuits with depth $\leq d$, size $\leq|\widehat{C}|^{O_{d}(\log |\widehat{C}|)}$, an additional $O_{d}\left(\log ^{2}|\widehat{C}|\right)$ arbitrary symmetric gates, and $|\widehat{C}|^{0.01}$ bits of output; $\epsilon=|\widehat{C}|^{-\Omega_{d}(\log |\widehat{C}|)}$.

3. If $\mathrm{TC}^{0} \neq \mathrm{NC}^{1}$ then for every $k$ and infinitely many $|C|, \mathcal{L}=\mathrm{TC}^{0}$ circuits with size $\leq|\widehat{C}|^{k}$ and $k \log |\widehat{C}|$ bits of output; $\epsilon=|\widehat{C}|^{-k}$.

4. $\mathcal{L}=\mathrm{AC}^{0}$ circuits with depth $\leq d$, size $\leq 2^{O_{d}\left(\mid \widehat{C}^{(1-\delta) / 3 d}\right)}$, and $|\widehat{C}|^{\delta / 3}$ bits of output, for any $\delta<1 ; \epsilon=2^{-\Omega_{d}\left(|\widehat{C}|^{(1-\delta) / 3 d}\right)}$.

Proof. For all four items, we choose $t=|C|$. As mentioned in $\S 1.1$, items 2-4 follow in a straightforward manner from Theorem 1.4 and the results of $\S 3$. Next we prove item 1 .

The key is to show that the local extension of any number-in-hand (NIH) protocol, under any partition satisfying a certain restriction discussed below, is computable by a numberon-forehead (NOF) protocol under a corresponding partition. This allows us to show that any NIH protocol breaking the security of $\widehat{C}$, in combination with the local reduction from 
Theorem 1.4, gives an NOF protocol that is not fooled by $\left(A_{t}\right)^{t}$ which contradicts item 1 of Theorem 1.5. We now give the details.

Let $F=\left\{f: G^{t} \rightarrow\{0,1\}^{|\widehat{C}|}\right\}$ denote the set of possible reductions from the proof of Theorem 1.4. Let $P$ be any $s$-party NOF partition of strings in $G^{t}$. We assign to each wire $j \in \widehat{C}$ a set $S_{j} \subseteq[s]$ of NOF players with the following property: for every $f \in F$ and $x \in G^{t}$, $j$ 's value in $f(x)$ depends only on elements of $x$ that are on the foreheads of players in $S_{j}$. Then if a given NIH partition $P^{\prime}$ of $\widehat{C}$ satisfies $\bigcup_{j \in P_{i}^{\prime}} S_{j} \subsetneq[s]$ for every set $P_{i}^{\prime} \in P^{\prime}$, we say that $P^{\prime}$ is simulatable. The key is that for any NIH protocol $\ell$ under a simulatable partition $P^{\prime}$ and any $f \in F, \ell(f(\cdot))$ is computable by an $s$-party NOF protocol under partition $P$ with the same amount of communication. Indeed, player $i$ 's communication in the NIH protocol can be simulated in the NOF protocol by any player in $[s] \backslash \bigcup_{j \in P_{i}^{\prime}} S_{j}$.

To construct our simulatable NIH partition, we note that the set of reductions $F$ given by the proof of Theorem 1.4 is the union of two sets $F_{1}$ and $F_{2}$, where $F_{i}$ contains the reductions arising from Claim $i . F_{1}$ and $F_{2}$ each contain $O(1)$-local reductions as previously discussed, but in fact they satisfy the stronger property that every bit $j$ in the output depends on the same $O(1)$ inputs for every function in the set. Formally, the following holds $\forall j \leq|\widehat{C}|$.

$$
\begin{aligned}
& \mid\left\{i \leq t \mid \exists f \in F_{1} \text { whose } j \text { th output depends on its } i \text { th input }\right\} \mid \leq 1 \\
& \mid\left\{i \leq t \mid \exists f \in F_{2} \text { whose } j \text { th output depends on its } i \text { th input }\right\} \mid \leq 4
\end{aligned}
$$

Now let $P$ be the partition of strings in $\left(A_{5}\right)^{t}$ onto $s=6$ foreheads given by Theorem 3.6. We define a simulatable partition $P^{\prime}$ of strings in $\{0,1\}^{|\widehat{C}|}$ into 6 hands as follows. For each wire $j \leq|\widehat{C}|$, we assign $j$ to hand $i$ for some $i \in[6]$ such that for every $f \in F_{1} \cup F_{2}$, the $j$ th output bit of $f$ does not depend on any input element on forehead $i$ in $P$. This is possible because each output bit depends on $\leq 4+1=5$ input elements and thus $\leq 5$ foreheads of $P$, and $P^{\prime}$ is simulatable because we have $S_{j} \subsetneq[6]$ for every $j$.

To complete the proof, recall that Theorem 1.4 shows that for every $k \in\{0,1\}^{n}$, if there is a function $\ell$ on domain $\{0,1\}^{|\widehat{C}|}$ and an $x \in\{0,1\}^{n}$ such that

$$
\Delta\left(\ell\left(\widehat{W}_{x}\right), \ell(S(C, x, \widehat{C}(x))) \geq \epsilon \cdot t \cdot|C|\right.
$$

for $\widehat{C} \leftarrow \operatorname{Comp}(C, k)$, then there exists $\alpha \in G$ and a function $f \in F_{1} \cup F_{2}$ such that

$$
\Delta\left(\ell\left(f\left(D_{\alpha}\right)\right), \ell\left(f\left(U_{G^{t}}\right)\right)\right) \geq \epsilon
$$

If (10) holds for a NIH protocol $\ell$ under $P^{\prime}$, then (11) contradicts item 1 of Theorem 1.5.

The previous proof constructed a simulatable NIH partition $P$ of $\widehat{C}$ into 6 sets each of size $O(|\widehat{C}|)=O\left(t^{2} \cdot|C| \cdot \log |G|\right)$. To analyze the security of our compiler in the only computation leaks (OCL) model, we now show that this partition can be refined into a simulatable, topologically-ordered partition with $O(t \cdot|C|)$ sets each of size $O(t \log |G|)$. (Recall from $\S 1.1$ that $P$ is topologically-ordered if for each $P_{i} \in P$ and each wire $j \in P_{i}, j$ 's value in $\widehat{C}$ 's computation depends only on wires $j^{\prime}$ such that $j^{\prime} \in P_{i^{\prime}}$ for some $i^{\prime} \leq i$.) In the following, the canonical partition of $x \in G^{t}$ for $s$-party NOF protocols is the one in which player $i$ 's forehead contains $x_{i}, x_{i+s}, \ldots, x_{i+t-s}$. 
Theorem 1.7. Assume that 8-party NOF protocols communicating $\leq c$ bits are $\epsilon$-fooled by $G^{t}$ under the canonical partition of $x \in G^{t}$.

Then for each $C$ and $k$ there is a topologically-ordered partition $P$ on $\widehat{C}:=\operatorname{Comp}(C, k)$ containing $O(t \cdot|C|)$ sets each of size $O(t \log |G|)$, such that Comp from Theorem 1.4 is an $(\mathcal{L}, \epsilon \cdot t \cdot|C|)$-leakage secure compiler for $\mathcal{L}=$ all $O C L$ leakage functions that output $\leq \delta \cdot c / t$ bits per set in $P$, where $\delta$ is a constant that depends only on the maximum fanout of $C$.

Proof. In the OCL model, the compiler specifies a topologically-ordered partition $P$ on the wires of the compiled circuit $\widehat{C}$, and the adversary adaptively chooses leakage functions to be applied to the wires of each set in $P$. We observe that any such adversary defines a number-in-hand (NIH) communication protocol on $\widehat{C}(x)$ 's wires under $P$. Thus following the proof above, any OCL adversary under a simultable partiton $P$ that breaks the security of $\widehat{C}$ contradicts item 1 of Theorem 1.5. (For this theorem we consider 8-party protocols rather than 6-party solely because it makes the partition cleaner to describe.)

Recall the two classes of reduction functions $f: G^{t} \rightarrow\{0,1\}^{|\widehat{C}|}$ in Theorem 1.4. In the first class, $f(x)$ plugs $x$ into the bundle $L$ in some multiplication tree of some RANDOM gadget, and reconstructs the other wires in the tree via the method described in Lemma 2.1; all wires outside this tree (and the gadget) are fixed in f's output by the hybrid argument.

In the second class, $f(x)$ plugs $x$ into a bundle at the output of some RANDOM gadget (thus also at the input of some NAND gadget). It then computes honestly the wires in the NAND gadget, reconstructs the RANDOM gadget that outputs $x$, and reconstructs the RANDOM gadgets that take the NAND gadget's output as input. All other wires are fixed in $f$ 's output by the hybrid argument.

We now describe the partition $P$ and the sets $S_{w} \subseteq[8]$ for each wire $w \in \widehat{C}$. (Recall that for $P$ to be simulatable, each $S_{w}$ must have the property that $w$ 's value in the output of any reduction $f(x)$ depends only on the foreheads of players in $S_{w}$ under the canonical partition of $x$.) $P$ first splits $\widehat{C}$ into $O(|C|)$ sets corresponding to the gadgets, and then refines each gadget's set as described below.

Partition for NAND. First let $w$ be a wire in a NAND gadget $N$. If $w$ is $N$ 's $i$ th input wire $(1 \leq i \leq t)$, then $S_{w}=\{i \bmod 8\}$. This is because for each $f \in F$, w's value in $f(x)$ 's output is either fixed by the hybrid argument or is equal to $x_{i}$ which is on the forehead of player $i \bmod 8$.

If $w$ is a wire at the output of an inversion gate whose input is N's ith input wire, then we again have $S_{w}=\{i \bmod 8\}$.

If $w$ is a wire at the output of a multiplication gate in $N$ (including $N$ 's output wires), we have either $S_{w}=\{1,2,3,4\}$ or $S_{w}=\{5,6,7,8\}$. This is because each multiplication gate depends on $\leq 4$ consecutive elements from $N$ 's input.

Thus we partition each NAND gadget into two sets of equal size $O(t \log |G|)$ : the wires $w$ such that $S_{w} \subseteq\{1,2,3,4\}$, and the wires $w$ such that $S_{w} \subseteq\{5,6,7,8\}$. Note that so far $P$ is topologically ordered and simulatable.

Partition for RANDOM. Now let $w$ be a wire in a RANDOM gadget $D$. If $w$ is $D$ 's $i$ th output wire then we have $S_{w}=\{i \bmod 8\}$ for the same reason as above: $w$ 's value is either fixed by the hybrid argument or is equal to $x_{i}$. 
If $w$ is one of $D$ 's input wires then by construction it is an output wire of some NAND gadget, and so we already have $S_{w}=\{1,2,3,4\}$ or $S_{w}=\{5,6,7,8\}$. Recall that each input wire of $D$ becomes the middle input to a $(2 t+1)$-wise multiplication tree, in which the leftmost $t$ inputs are denoted $L$ and the rightmost $t$ inputs are denoted $R$.

If $w$ is the $i$ th wire $(1 \leq i \leq t)$ in a bundle $L$ of some tree, then we have $S_{w}=\{i \bmod 8\}$ because again $w$ 's value is either fixed by the hybrid argument or is equal to $x_{i}$.

If $w$ is the $i$ th wire in a bundle $R$ of some tree, then by the reconstruction procedure in Lemma $2.1 w$ depends only on its "mirror image" in $L$, i.e. the $(t-i+1)$ th wire of $L$; thus we have $S_{w}=\{t-i+1 \bmod 8\}$. There are two exceptions to this. First, the first wire of $R$ also depends on the middle input wire of the tree, i.e. one of $D$ 's input wires (recall that this dependence is in the reconstruction procedure, not in the gadget's computation itself); thus for this wire we have either $S_{w}=\{1,2,3,4,8\}$ or $S_{w}=\{5,6,7,8\}$. Second, the last wire of $R$ also depends on the output wire of this tree, i.e. one of $D$ 's output wires; thus for this wire we have $S_{w}=\{1, m\}$ for some $m \in[8]$.

We now illustrate the sets $S_{w}$ for the $2 t+1$ input wires of a multiplication tree in $D$. Either they are

$$
\overbrace{\{1\} \cdots\{8\} \ldots\{1\} \cdots\{8\}}^{L}\{1,2,3,4\} \overbrace{\{1,2,3,4,8\}\{7\} \cdots\{1\} \cdots\{8\} \cdots\{2\}\{1, m\}}^{R}
$$

for some $m \in[8]$, or else they are

$$
\overbrace{\{1\} \cdots\{8\} \ldots\{1\} \cdots\{8\}}^{L}\{5,6,7,8\} \overbrace{\{5,6,7,8\}\{7\} \cdots\{1\} \cdots\{8\} \cdots\{2\}\{1, m\}}^{R}
$$

$P$ partitions each such set of input wires as follows, which induces a partition on the whole tree. Essentially, $P$ works from the outside towards the middle, grouping blocks of 4 consecutive wires from each side. So, the first set contains wires $1, \ldots, 4$ of $L$ and wires $(t-3), \ldots, t$ of $R$, the next set contains wires $5, \ldots, 8$ of $L$ and wires $(t-7), \ldots,(t-4)$ of $R$, and so on. The middle $8+1+8=17$ wires are handled differently, but note that for each set so far we have $\bigcup_{w} S_{w}=\{5,6,7,8\}$ or $\bigcup_{w} S_{w}=\{1,2,3,4, m\}$ for some $m \in[8]$.

We partition the middle 17 wires into two sets as follows. If the middle wire has $S_{w}=$ $\{5,6,7,8\}$ then we partition as the other wires: the outermost 8 wires form one set (with $\bigcup_{w} S_{w}=\{1,2,3,4\}$ ), and the innermost 9 form the other (with $\bigcup_{w} S_{w}=\{5,6,7,8\}$ ). If instead the middle wire has $S_{w}=\{1,2,3,4\}$, then we make one set from the outermost 10 wires (with $\bigcup_{w} S_{w}=\{1,2,3,4,5\}$ ), and the other from the innermost 7 wires (with $\left.\bigcup_{w} S_{w}=\{1,2,3,4,6,7,8\}\right)$.

The partition on the input wires induces a partition on the whole tree in the natural way: each internal wire, which is the output of some multiplication gate, is assigned to the set containing the input wire on which it depends. This preserves the value of each $\bigcup_{w} S_{w}$ listed above, and thus the partition is simulatable. It is also topologically ordered, as the sets in the partition form "concentric subtrees" that can be evaluated from the inside out.

For each tree, we have created a partition into $O(t)$ sets each of size $O(\log |G|)$. (Here the hidden constants depend on $s=O(1)$.) Since there are $t$ trees in a RANDOM gadget, naïvely this partitions the whole gadget into $O\left(t^{2}\right)$ sets each of size $O(\log |G|)$. However, by combining the sets from each tree that are at the same depth in the concentric subtrees, we 
can instead get a partition into $O(t)$ sets each of size $O(t \log |G|)$. This partition remains simulatable and topologically ordered.

Overall, we have $O(t \cdot|C|)$ sets each of size $O(t \log |G|)$ as promised.

From NIH to NOF. Assume that, for some circuit $C$ and string $k$, there is an OCL adversary $\ell$ under partition $P$ and an input $x$ such that $\Delta\left(\ell\left(\widehat{W}_{x}\right), \ell(S(C, x, \widehat{C}(x))) \geq \epsilon \cdot t \cdot|C|\right.$, where $\widehat{C} \leftarrow \operatorname{Comp}(C, k)$ and $S$ is the simulator from Theorem 1.4. Then by Theorem 1.4 there is some $\alpha \in G$ and $f \in F$ such that $\Delta\left(\ell\left(f\left(D_{\alpha}\right)\right), \ell\left(f\left(U_{G^{t}}\right)\right)\right) \geq \epsilon$. By construction, the function $\ell(f(x))$ can be computed by an $s$-party NOF protocol under the canonical partition of $x \in G^{t}$, because $\ell$ corresponds to an NIH protocol and $P$ is simulatable. The only remaining question is how much communication is required in the NOF protocol.

Let $b \in \mathbb{N}$ be a bound on the output length of each leakage function chosen by the OCL adversary, i.e. a bound on the amount of communication by each player in the NIH protocol. Let $d$ denote the maximum fanout of any gate in $C$. The key point is that for each $f \in F$, all wire values in $f(x) \in\{0,1\}^{|\widehat{C}|}$ are fixed by the hybrid argument (independent of $x$ ) except for in at most $d+O(1)$ gadgets, and thus in at most $O(t d)$ sets in $P$. Since no communication is needed to simulate the NIH players whose inputs are fixed, the NOF protocol communicates $O(t d b)$ bits in total. Thus if $b=\delta c / t$ for sufficiently small $\delta$ that depends only on $d$, this contradicts the assumption of the theorem.

\section{Multi-query security via secure hardware}

In this section we consider the extension of our construction to the setting where the adversary can make multiple, adaptive queries. We follow the approach of $\left[\mathrm{FRR}^{+} 10\right]$, using a simple secure hardware component. As mentioned in $§ 1.1$, in our setting this component has no input and outputs a sample from the distribution $D_{\text {id }}$.

We generalize Definition 1.1 to the multi-query setting following [FRR ${ }^{+} 10$, Definition 1], beginning with an overview. As before, the adversary $A$ is restricted to choosing leakage functions from some class $\mathcal{L}$ and remains otherwise computationally unbounded. $A$ makes $q$ queries to the circuit $\widehat{C}$, denoted $\left(x_{i}, \ell_{i}\right) \in\{0,1\}^{n} \times \mathcal{L}$ for $i \leq q$, and in response to the $i$ th query $A$ receives $\left(\widehat{C}\left(x_{i}\right), \ell_{i}\left(\widehat{W}_{i}\right)\right)$ where $\widehat{W}_{i}$ denotes the wires of $\widehat{C}\left(x_{i}\right) . A$ chooses its queries adaptively, meaning that each query can depend on all responses seen so far.

On input $(C, k)$, the compiler from $\S 2$ implicitly computes a string $\widehat{k}_{0} \in\left(G^{t}\right)^{n}$ that encodes $k$. In the multi-query setting, this encoding must be "refreshed" between queries, as otherwise $A$ can learn, say, the first bit of $k$ after $O(t)$ queries. To accomplish this, the compiler below outputs a circuit $\widehat{C}:\{0,1\}^{n} \times\left(G^{t}\right)^{n} \rightarrow\{0,1\}^{n} \times\left(G^{t}\right)^{n}$ as well as an initial encoding $\widehat{k}_{0}$. Then, the second output $\widehat{k}_{i}$ of $\widehat{C}\left(x_{i}, \widehat{k}_{i-1}\right)$ is used as the second input to $\widehat{C}$ in the $(i+1)$ th query. (This corresponds to the notion of a stateful circuit in [FRR 10$]$.) Crucially $A$ does not directly obtain any $\widehat{k}_{i}$, but the leakage functions operate on these values as they are carried on wires of $\widehat{C}$.

For an adversary $A$ interacting with such a circuit $\widehat{C}$ on initial encoding $\widehat{k}_{0}$, we let 
$\left(x_{1}, \ell_{1}\right)=A(\widehat{C})$ be the first query, and then inductively define

$$
\begin{aligned}
\left(y_{i}, \widehat{k}_{i}\right) & :=\widehat{C}\left(x_{i}, \widehat{k}_{i-1}\right) \\
\left(x_{i+1}, \ell_{i+1}\right) & :=A\left(\widehat{C}, x_{1}, y_{1}, \ell_{1}\left(\widehat{W}_{1}\right), \ldots, x_{i}, y_{i}, \ell_{i}\left(\widehat{W}_{i}\right)\right) .
\end{aligned}
$$

We note two final differences from the single-query setting. First, the circuit $\widehat{C}$ is randomized, which means that it contains gates whose output comes from some distribution rather than being deterministically fixed by the input. (In our construction, the only randomized gates will be the secure hardware components which output a sample from $D_{\text {id.) }}$ Second, the simulator $S$ is stateful, which means that between subsequent evaluations it maintains some state. (In our construction the state will be an element of $\left(G^{t}\right)^{n}$.)

Definition 5.1. Let $\operatorname{Comp}(\cdot, \cdot)$ be a randomized algorithm that takes as input a circuit $C:\{0,1\}^{n} \times\{0,1\}^{n} \rightarrow\{0,1\}^{n}$ and a string $k \in\{0,1\}^{n}$. For a set of functions $\mathcal{L}$, Comp is a $q$-query $(\mathcal{L}, \epsilon)$-leakage-secure compiler if the following properties hold.

1. (Structure.) For every $C$ and $k, \operatorname{Comp}(C, k)$ outputs a string $\widehat{k}_{0} \in\left(G^{t}\right)^{n}$, and a randomized circuit $\widehat{C}:\{0,1\}^{n} \times\left(G^{t}\right)^{n} \rightarrow\{0,1\}^{n} \times\left(G^{t}\right)^{n}$ which is completely determined by $C$.

2. (Correctness.) For every $A$ as above, every $C, k$, and every $i \leq q$ : $y_{i}=C\left(x_{i}, k\right)$ with probability 1.

3. (Security.) There is a randomized polynomial-time stateful algorithm $S$ such that the following holds for every $C, k$ and every $A$ as above. Let $D_{\text {real }}$ denote the distribution $\left(\ell_{1}\left(\widehat{W}_{1}\right), \ldots, \ell_{q}\left(\widehat{W}_{q}\right)\right)$, and let $D_{\text {sim }}$ denote the corresponding distribution $\left(\ell_{1}\left(S\left(C, x_{1}, y_{1}\right)\right), \ldots, \ell_{q}\left(S\left(C, x_{q}, y_{q}\right)\right)\right)$ when each $\widehat{W}_{j}$ in $(12)(1 \leq j \leq i)$ is replaced with $S\left(C, x_{j}, y_{j}\right)$. Then, $\Delta\left(D_{\text {real }}, D_{\text {sim }}\right) \leq \epsilon$.

The construction. Recall from $\S 1.1$ that a $D_{\text {id }}$-gate is a gate with no input that on each execution of the circuit outputs a string of length $2 t$ sampled from $D_{\text {id }}$, and any circuit that contains one or more $D_{\text {id }}$-gates is a $D_{\text {id }}$-circuit.

Then, Comp outputs a $D_{\text {id-circuit }} \widehat{C}:\{0,1\}^{n} \times\left(G^{t}\right)^{n} \rightarrow\{0,1\}^{n} \times\left(G^{t}\right)^{n}$ as follows. $\widehat{C}$ is identical to the construction in $\S 2$ with two exceptions. First, each pair $\left(R^{(i)}, L^{(i+1)}\right)$ in each RANDOM gadget is computed by a $D_{\text {id }}$-gate. Second, $\widehat{C}$ computes its second output $\widehat{k}_{i}$ by applying a RANDOM gadget to each bundle of its second input $\widehat{k}_{i-1}$.

The simulator $S$ then operates as follows. For the first query, $S$ chooses $\widehat{k}_{0}, \widehat{k}_{1} \in\left(G^{t}\right)^{n}$ uniformly at random, and produces wire values for $\widehat{C}\left(x_{1}, \widehat{k}_{0}\right)$ conditioned on output $\left(y_{1}, \widehat{k}_{1}\right)$ as described in Theorem 1.4. Between queries $i$ and $i+1, S$ stores the value $\widehat{k}_{i}$, and for the $(i+1)$ th query it chooses $\widehat{k}_{i+1}$ uniformly at random and proceeds in the same manner.

Proving the security of this construction requires a slightly stronger property of the group encoding than what is given by Definition 1.2. Namely, it requires that the leakage class $\mathcal{L}$ cannot distinguish the distributions $D_{\alpha}$ and $U_{G^{t}}$ even with two adaptive queries. The need for this is due to the fact that each $\widehat{k}_{i}(1 \leq i<q)$ is given as input to two leakage functions: once when $\widehat{k}_{i}$ is an output of $\widehat{C}$ and once when it is an input. Formally, we require the following strengthening of Definition 1.2. 
Definition 5.2. Let $G$ be a group and $t \in \mathbb{N}$. A set of functions $\mathcal{L}$ is 2-adaptive $\epsilon$-fooled by $G^{t}$ if for every $\alpha \in G$, every $\ell \in \mathcal{L}$, and every function $A$ : range $(\ell) \rightarrow \mathcal{L}$, the following two distributions are $\epsilon$-close in statistical distance.

1. Sample $w \leftarrow D_{\alpha}$, compute $\ell^{\prime}:=A(\ell(w))$, and output $\left(\ell(w), \ell^{\prime}(w)\right)$.

2. Sample $w \leftarrow U_{G^{t}}$, compute $\ell^{\prime}:=A(\ell(w))$, and output $\left(\ell(w), \ell^{\prime}(w)\right)$.

With this stronger property, the following theorem can be proved by building on Theorem 1.4. Specifically, one first uses Theorem 1.4 to show that the security property of Definition 5.1 holds when the simulator chooses each $\widehat{k}_{i}$ as in the real execution but the internal wires of each evaluation $\widehat{C}\left(x_{i}, \widehat{k}_{i}\right)$ are reconstructed. Then, a hybrid argument over each bundle in each $\widehat{k}_{i}$ is used to show that the security property is satisfied even when $S$ chooses each $\widehat{k}_{i}$ uniformly at random. The proof is essentially identical to the proof of $\left[\mathrm{FRR}^{+} 10\right.$, Lemma $15]$, and we omit the details.

Theorem 5.3. Let $G$ be a group. For every polynomial-time computable function $t=$ $t(n,|C|)$, there is a compiler Comp for which the following holds.

1. For every $C:\{0,1\}^{n} \times\{0,1\}^{n} \rightarrow\{0,1\}^{n}$ and $k \in\{0,1\}^{n}, \operatorname{Comp}(C, k)$ runs in time poly $(|C|, t)$ and outputs a $D_{\text {id }}$-circuit $\widehat{C}$ of size $O\left(t^{2} \cdot|C|\right)$ and depth $O(t \cdot \operatorname{depth}(C))$.

2. For every set of functions $\mathcal{L}$, every $q \in \mathbb{N}$, and every $\epsilon>0$, if the 4-local extension of $\mathcal{L}$ is 2-adaptive $\epsilon$-fooled by $G^{t}$ then Comp is a q-query $\left(\mathcal{L}, \epsilon^{\prime}\right)$-leakage-secure compiler for $\epsilon^{\prime}:=(q+1) \cdot \epsilon \cdot(n+t \cdot|C|)$.

Similarly to Corollary 1.6, Corollary 1.8 is derived as a result of Theorem 5.3 and the following subsection, by choosing $t=|C|$.

\subsection{Adaptive compression bounds}

In this section, we show that the function classes from $\S 3$ are 2-adaptive fooled by $\left(A_{5}\right)^{t}$. For the class of functions computable by $\mathrm{AC}^{0}$ with symmetric gates, this requires asymptotically smaller output length.

\subsubsection{Multi-party protocols}

Theorem 5.4. There is a partition of the inputs in $\left(A_{5}\right)^{t}$ into $k$ pieces such that the set $\mathcal{L}$ of $k$-party number-on-forehead protocols communicating $c$ bits and outputting $\leq c$ bits is 2-adaptive $\epsilon$-fooled by $\left(A_{5}\right)^{t}$ for $\epsilon=2^{c-\Omega\left(t /\left(k^{2} 4^{k}\right)\right)}$.

Proof. The partition is the same as in Theorem 3.6. Assume that $\mathcal{L}$ is not 2-adaptive $\epsilon$-fooled by $\left(A_{5}\right)^{t}$, and let $\alpha \in A_{5}, P^{\prime} \in \mathcal{L}$, and $A: \operatorname{range}\left(P^{\prime}\right) \rightarrow \mathcal{L}$ violate Definition 5.2.

Consider the following $k$-party protocol $P$ that communicates and outputs $2 c$ bits. On input $x$, the parties first compute $P^{\prime}(x)$ by communicating $c$ bits. Then, they each determine $P^{\prime \prime}:=A\left(P^{\prime}(x)\right)$ with no communication. Finally, they compute $P^{\prime \prime}(x)$ again communicating $c$ bits. By assumption we have $\Delta\left(P\left(D_{\alpha}\right), P\left(U_{\left(A_{5}\right)^{t}}\right)\right) \geq \epsilon$, which contradicts Theorem 3.6. 


\subsection{2 $\mathrm{TC}^{0}$}

Theorem 5.5. If $\mathrm{TC}^{0} \neq \mathrm{NC}^{1}$ then $\forall k$ and infinitely many $t$, the class $\mathcal{L}$ of $\mathrm{TC}^{0}$ circuits with size $\leq t^{k}$ and output length $k \log t$ is 2-adaptive $t^{-k}$-fooled by $\left(A_{5}\right)^{t}$.

Proof. Assume that there exists $k$ such that for sufficiently large $t, \mathcal{L}$ is not 2-adaptive $t^{-k}$ fooled by $\left(A_{5}\right)^{t}$. Let $\alpha \in A_{5}, \ell:\left(A_{5}\right)^{t} \rightarrow\{0,1\}^{k \log t}$, and $A:\{0,1\}^{k \log t} \rightarrow \mathcal{L}$ be the choices that violate Definition 5.2. Let $\ell_{1}^{\prime}, \ldots, \ell_{t^{k}}^{\prime} \in \mathcal{L}$ be all possible circuits that $A$ could output.

Let $\ell^{\prime \prime}:\left(A_{5}\right)^{t} \rightarrow\{0,1\}^{2 k \log t}$ be the following procedure. On input $x \in\left(A_{5}\right)^{t}$ : first compute $\ell(x)$, then select $\ell^{\prime}:=\ell_{\ell(x)}^{\prime}$ (identifying $\ell(x) \in\{0,1\}^{k \log t}$ with the natural number it represents), and finally output $\left(\ell(x), \ell^{\prime}(x)\right)$. Clearly $\ell^{\prime \prime}$ is computable by a $\mathrm{TC}^{0}$ circuit of size $t^{O(k)}$, and by assumption we have $\Delta\left(\ell^{\prime \prime}\left(D_{\alpha}\right), \ell^{\prime \prime}\left(U_{\left(A_{5}\right)^{t}}\right)\right) \geq t^{-k}$ which contradicts Theorem 3.9 .

\subsection{3 $\mathrm{AC}^{0}$}

The proof of the following theorem is essentially identical to the previous proof.

Theorem 5.6. For every $0<\delta<1$ and every integer $d>0$, there is a constant $\epsilon>0$ such that the following holds. Let $\mathcal{L}$ be the class of unbounded-fanin circuits $C:\left(A_{5}\right)^{t} \rightarrow\{0,1\}^{t^{\delta}}$ of depth $\leq d$ and size $\leq 2^{\epsilon \cdot t^{(1-\delta) / d}}$. Then, $\mathcal{L}$ is 2-adaptive $2^{-\epsilon \cdot t^{(1-\delta) / d}}$-fooled by $\left(A_{5}\right)^{t}$.

Proof. Assume that $\mathcal{L}$ is not 2-adaptive $2^{-\epsilon \cdot t^{(1-\delta) / d}}$-fooled by $\left(A_{5}\right)^{t}$, and let $\alpha \in A_{5}, \ell \in \mathcal{L}$, and $A:\{0,1\}^{t^{\delta}} \rightarrow \mathcal{L}$ be the choices that violate Definition 5.2 . Let $\ell_{1}^{\prime}, \ldots, \ell_{2^{t^{\delta}}}^{\prime} \in \mathcal{L}$ be all possible circuits that $A$ could output.

Let $\ell^{\prime \prime}:\left(A_{5}\right)^{t} \rightarrow\{0,1\}^{2 t^{\delta}}$ be the following procedure. On input $x \in\left(A_{5}\right)^{t}$ : first compute $\ell(x)$, then select $\ell^{\prime}:=\ell_{\ell(x)}^{\prime}$, and finally output $\left(\ell(x), \ell^{\prime}(x)\right)$. Clearly $\ell^{\prime \prime}$ is computable by an $\mathrm{AC}^{0}$ circuit of depth $2 d+O(1)$ and size $2 \cdot 2^{\epsilon \cdot t^{(1-\delta) / d}}+2^{t^{\delta}}+O(1)=2^{\epsilon^{\prime} \cdot t^{\left(1-\delta^{\prime}\right) /(2 d+O(1))}}$ for an appropriate $\epsilon^{\prime}$ and $\delta^{\prime}$, which contradicts Theorem 3.11 .

\subsection{4 $\mathrm{AC}^{0}$ with symmetric gates}

Recall that in $\S 3.4$, it was shown that $\left(A_{5}\right)^{t} t^{-\Omega(\log t)}$-fools the class of unbounded-fan-in constant-depth circuits that contain $t^{O(\log t)}$ And/Or/Not gates and $O\left(\log ^{2} t\right)$ arbitrary symmetric gates and output $t^{0.1}$ bits. To apply the technique from the two preceding proofs to this class, one needs to restrict the output length to $O(1)$ to ensure that the circuit $\ell^{\prime \prime}$ still contains only $O\left(\log ^{2} t\right)$ symmetric gates. However, by a more careful extension of Theorem 3.12 we can improve the output length to $\Omega\left(\log ^{2} t\right)$. In the following, we focus mainly on the necessary changes to the proof of Theorem 3.12 .

Theorem 5.7. For every $d$, there is an $\epsilon>0$ such that the following holds for every $t$.

Let $\mathcal{L}$ be the set of functions $\ell:\left(A_{5}\right)^{t} \rightarrow\{0,1\}^{\epsilon \log ^{2} t}$ where each output bit of $\ell$ is computable by an unbounded-fan-in circuit of depth $\leq d$ that contains $\leq t^{\epsilon \log t}$ And/Or/Not gates and $\leq \epsilon \log ^{2} t$ arbitrary symmetric gates.

Then, $\mathcal{L}$ is 2-adaptive $t^{-\epsilon \log t}$-fooled by $\left(A_{5}\right)^{t}$. 
Proof. Assume that $\mathcal{L}$ is not 2-adaptive $t^{-\epsilon \log t}$-fooled by $\left(A_{5}\right)^{t}$, and let $\alpha \in A_{5}, \ell_{0} \in \mathcal{L}$, and $A:\{0,1\}^{\epsilon \log ^{2} t} \rightarrow \mathcal{L}$ be the choices that violate Definition 5.2. Let $\ell_{1}, \ldots, \ell_{t^{\epsilon \log t}} \in \mathcal{L}$ be all possible circuits that $A$ could output.

For an appropriate $n=\Omega(\sqrt{t} / \log t)$ and for each $i=0, \ldots, t^{\epsilon \log t}$, let $\ell_{i}^{\prime}:\{0,1\}^{n^{2} \cdot 0.3 \log n} \rightarrow$ $\{0,1\}^{\epsilon \log ^{2} t}$ be the corresponding function given by Lemma 3.14. These functions have the property that the distribution $\left(\ell_{0}^{\prime}(x), \ell_{\ell_{0}^{\prime}(x)}^{\prime}(x)\right)$ when $x \leftarrow P A P^{-1}(1)$ has statistical distance $\geq n^{-\epsilon^{\prime} \log n}$ from the corresponding distribution when $x \leftarrow P A P^{-1}(0)$, for $\epsilon^{\prime}=\epsilon^{\prime}(\epsilon)>0$.

Let $R$ be the distribution on random restrictions $\rho$ given in the proof of Theorem 3.12. Say that $\rho$ is $\operatorname{good}$ if $\left.P A P\right|_{\rho}=G I P$ (i.e. $\left.P A P\right|_{\rho}$ has exactly 1 input restricted per bottom $\oplus$ gate) and for every $i \leq t^{\epsilon \log t}$ and $j \leq \epsilon \log ^{2} t,\left.\ell_{i, j}^{\prime}\right|_{\rho}$ is computable by a $(0.3 \log n)$-party protocol exchanging $\log ^{5} n$ bits (where $\ell_{i, j}^{\prime}$ denotes the $j$ th output bit of $\ell_{i}^{\prime}$.) Because the number of $\ell_{i, j}^{\prime}$ is $\epsilon \log ^{2} t \cdot\left(t^{\epsilon \log t}+1\right)$ and because $t=n^{O(1)}$, when $\epsilon$ is sufficiently small we obtain

$$
\operatorname{Pr}_{\rho \leftarrow R}[\rho \text { is good }] \geq 1-n^{\Omega(\log n)}
$$

by combining [Vio07, Claim $11 \&$ Lemma 12] with a union bound.

For any $\rho$ that is good, let $P_{\rho}:\{0,1\}^{n \cdot 0.3 \log n} \rightarrow\{0,1\}$ be the following $(0.3 \log n)$-party protocol exchanging $2 \epsilon \log ^{2} t \log ^{5} n+1=\log ^{O(1)} n$ bits. On input $y$, the parties first compute each of the $\epsilon \log ^{2} t$ output bits of $\left.\ell_{0}^{\prime}\right|_{\rho}(y)$, exchanging a total of $\epsilon \log ^{2} t \log ^{5} n$ bits. Then, each party chooses $\ell_{i}^{\prime}:=\ell_{\ell_{0}^{\prime} \mid \rho(y)}^{\prime}$ with no communication. Then, the parties compute each of the $\epsilon \log ^{2} t$ output bits of $\left.\ell_{i}^{\prime}\right|_{\rho}(y)$ again exchanging $\epsilon \log ^{2} t \log ^{5} n$ bits. Finally, the parties use one additional bit of communication to output 1 iff $\left(\left.\ell_{0}^{\prime}\right|_{\rho}(y),\left.\ell_{i}^{\prime}\right|_{\rho}(y)\right) \in S$ for the appropriate set $S$ corresponding to (8) in Theorem 3.12.

The rest of the proof follows the same argument as Theorem 3.12.

\section{References}

[AAW10] Eric Allender, Vikraman Arvind, and Fengming Wang. Uniform derandomization from pathetic lower bounds. In Workshop on Randomization and Computation (RANDOM), pages $380-393,2010$.

[AIK06] Benny Applebaum, Yuval Ishai, and Eyal Kushilevitz. Cryptography in $\mathrm{NC}^{0}$. SIAM J. on Computing, 36(4):845-888, 2006.

[All01] Eric Allender. The division breakthroughs. Bulletin of the EATCS, 74:61-77, 2001.

[Bar89] David A. Mix Barrington. Bounded-width polynomial-size branching programs recognize exactly those languages in $\mathrm{NC}^{1}$. J. of Computer and System Sciences, 38(1):150$164,1989$.

$\left[\mathrm{BGI}^{+} 01\right]$ Boaz Barak, Oded Goldreich, Russell Impagliazzo, Steven Rudich, Amit Sahai, Salil P. Vadhan, and Ke Yang. On the (im)possibility of obfuscating programs. In Int. Cryptology Conf. (CRYPTO), pages 1-18, 2001.

[BNS92] László Babai, Noam Nisan, and Márió Szegedy. Multiparty protocols, pseudorandom generators for logspace, and time-space trade-offs. J. of Computer and System Sciences, 45(2):204-232, 1992. 
[CFL83] Ashok K. Chandra, Merrick L. Furst, and Richard J. Lipton. Multi-party protocols. In 15th ACM Symp. on the Theory of Computing (STOC), pages 94-99, 1983.

[CL94] Jin-yi Cai and Richard J. Lipton. Subquadratic simulations of balanced formulae by branching programs. SIAM J. on Computing, 23(3):563-572, 1994.

[Cle91] Richard Cleve. Towards optimal simulations of formulas by bounded-width programs. Computational Complexity, 1:91-105, 1991.

[CM87] Stephen A. Cook and Pierre McKenzie. Problems complete for deterministic logarithmic space. J. Algorithms, 8(3):385-394, 1987.

[CT93] Fan R. K. Chung and Prasad Tetali. Communication complexity and quasi randomness. SIAM J. Discrete Math., 6(1):110-123, 1993.

[DF12] Stefan Dziembowski and Sebastian Faust. Leakage-resilient circuits without computational assumptions. In Theory of Cryptography Conf. (TCC), pages 230-247, 2012.

[DI06] Bella Dubrov and Yuval Ishai. On the randomness complexity of efficient sampling. In 38th ACM Symposium on Theory of Computing (STOC), pages 711-720, 2006.

[Dru12] Andrew Drucker. New limits to classical and quantum instance compression. In IEEE Symp. on Foundations of Computer Science (FOCS), 2012.

[FRR $\left.{ }^{+} 10\right]$ Sebastian Faust, Tal Rabin, Leonid Reyzin, Eran Tromer, and Vinod Vaikuntanathan. Protecting circuits from leakage: the computationally-bounded and noisy cases. In Int. Conf. on the Theory and Applications of Cryptographic Techniques (EUROCRYPT), pages 135-156, 2010.

[GGH ${ }^{+}$08] Shafi Goldwasser, Dan Gutfreund, Alexander Healy, Tali Kaufman, and Guy Rothblum. A (de)constructive approach to program checking. In 40th ACM Symposium on Theory of Computing (STOC), pages 143-152, 2008.

[GR10] Shafi Goldwasser and Guy N. Rothblum. Securing computation against continuous leakage. In Int. Cryptology Conf. (CRYPTO), pages 59-79, 2010.

[GR12] Shafi Goldwasser and Guy N. Rothblum. How to compute in the presence of leakage. In IEEE Symp. on Foundations of Computer Science (FOCS), 2012.

[HN10] Danny Harnik and Moni Naor. On the compressibility of NP instances and cryptographic applications. SIAM J. on Computing, 39(5):1667-1713, 2010.

[HP89] Derek Holt and W. Plesken. Perfect Groups. Oxford Mathematical Monographs. Clarendon Press, 1989.

[ISW03] Yuval Ishai, Amit Sahai, and David Wagner. Private circuits: Securing hardware against probing attacks. In Int. Cryptology Conf. (CRYPTO), pages 463-481, 2003.

[JV10] Ali Juma and Yevgeniy Vahlis. Protecting cryptographic keys against continual leakage. In Int. Cryptology Conf. (CRYPTO), pages 41-58, 2010.

[Kil88] Joe Kilian. Founding cryptography on oblivious transfer. In ACM Symp. on the Theory of Computing (STOC), pages 20-31, 1988. 
[KS04] Hans Kurzweil and Bernd Stellmacher. The Theory of Finite Groups: An Introduction. Springer, 2004.

[Mil14] Eric Miles. Iterated group products and leakage resilience against $\mathrm{NC}^{1}$. In ACM Innovations in Theoretical Computer Science conf. (ITCS), 2014.

[MR04] Silvio Micali and Leonid Reyzin. Physically observable cryptography. In Theory of Cryptography Conf. (TCC), pages 278-296, 2004.

[Nis93] Noam Nisan. The communication complexity of threshold gates. In Combinatorics, Paul Erdös is Eighty, number 1 in Bolyai Society Mathematical Studies, pages 301-315, 1993.

[Raz00] Ran Raz. The BNS-Chung criterion for multi-party communication complexity. Comput. Complexity, 9(2):113-122, 2000.

[Rot12] Guy N. Rothblum. How to compute under $\mathrm{AC}^{0}$ leakage without secure hardware. In Int. Cryptology Conf. (CRYPTO), pages 552-569, 2012.

[Vio07] Emanuele Viola. Pseudorandom bits for constant-depth circuits with few arbitrary symmetric gates. SIAM J. on Computing, 36(5):1387-1403, 2007.

[Vio13] Emanuele Viola. The communication complexity of addition. In ACM-SIAM Symp. on Discrete Algorithms (SODA), 2013.

[VW08] Emanuele Viola and Avi Wigderson. Norms, XOR lemmas, and lower bounds for GF(2) polynomials and multiparty protocols. Theory of Computing, 4:137-168, 2008. 